University of Nebraska - Lincoln

DigitalCommons@University of Nebraska - Lincoln

Faculty Publications from the Department of Electrical \& Computer Engineering, Department Electrical and Computer Engineering

$3-2012$

\title{
Transmission Strategies in Multiple-Access Fading Channels With Statistical QoS Constraints
}

Deli Qiao

University of Nebraska-Lincoln, dlqiao@ce.ecnu.edu.cn

M. Cenk Gursoy

University of Nebraska-Lincoln, gursoy@engr.unl.edu

Senem Velipasalar

University of Nebraska-Lincoln, velipasa@engr.unl.edu

Follow this and additional works at: https://digitalcommons.unl.edu/electricalengineeringfacpub

Part of the Electrical and Computer Engineering Commons

Qiao, Deli; Cenk Gursoy, M.; and Velipasalar, Senem, "Transmission Strategies in Multiple-Access Fading Channels With Statistical QoS Constraints" (2012). Faculty Publications from the Department of Electrical and Computer Engineering. 188.

https://digitalcommons.unl.edu/electricalengineeringfacpub/188

This Article is brought to you for free and open access by the Electrical \& Computer Engineering, Department of at DigitalCommons@University of Nebraska - Lincoln. It has been accepted for inclusion in Faculty Publications from the Department of Electrical and Computer Engineering by an authorized administrator of DigitalCommons@University of Nebraska - Lincoln. 


\title{
Transmission Strategies in Multiple-Access Fading Channels With Statistical QoS Constraints
}

\author{
Deli Qiao, Mustafa Cenk Gursoy, and Senem Velipasalar
}

\begin{abstract}
Effective capacity, which provides the maximum constant arrival rate that a given service process can support while satisfying statistical queueing constraints, is analyzed in a multiuser scenario. In particular, the effective capacity region of fading multiple-access channels in the presence of quality of service (QoS) constraints is studied. Perfect channel side information is assumed to be available at both the transmitters and the receiver. It is initially assumed that the transmitters send the information at a fixed power level and, hence, do not employ power control policies. Under this assumption, the performance achieved by superposition coding with successive decoding techniques is investigated. It is shown that varying the decoding order with respect to the channel states can significantly increase the achievable throughput region. In the two-user case, the optimal decoding strategy is determined for the scenario in which the users have the same QoS constraints. The performance of orthogonal transmission strategies is also analyzed. It is shown that for certain QoS constraints, time-division multiple access can achieve better performance than superposition coding if fixed successive decoding order is used at the receiver side. In the subsequent analysis, power control policies are incorporated into the transmission strategies. The optimal power allocation policies for any fixed decoding order over all channel states are identified. For a given variable decoding-order strategy, the conditions that the optimal power control policies must satisfy are determined, and an algorithm that can be used to compute these optimal policies is provided.
\end{abstract}

Index Terms-Effective capacity, multiple-access fading channels, power control, queueing constraints, successive decoding, superposition coding, time-division multiple access (TDMA).

\section{INTRODUCTION}

$\mathbf{I}$ $\mathrm{N}$ wireless networks, the design and analysis of efficient transmissions strategies have been of significant interest for many years. In particular, fading multiple-access channels (MAC) have been extensively studied from an information-theoretic point of view [1]-[7]. For instance, Tse and Hanly [3] have characterized the capacity region of and determined the

Manuscript received September 10, 2010; revised March 07, 2011; accepted July 29, 2011. Date of publication November 11, 2011; date of current version February 29,2012. The material in this paper was presented in part at the 2010 IEEE International Conference on Communications. This work was supported by the National Science Foundation under Grants CCF-0546384 (CAREER), CNS-0834753, and CCF-0917265.

D. Qiao is with the Department of Electrical Engineering, University of Nebraska-Lincoln, Lincoln, NE68588 USA (e-mail: dqiao726@huskers.unl.edu).

M. C. Gursoy and S. Velipasalar are with the Department of Electrical Engineering and Computer Science, Syracuse University, Syracuse, NY 13244 USA (e-mail: mcgursoy@syr.edu; svelipas@syr.edu).

Communicated by R. Berry, Associate Editor for Communications.

Color versions of one or more of the figures in this paper are available online at http://ieeexplore.ieee.org.

Digital Object Identifier 10.1109/TIT.2011.2175900 optimal resource allocation policies for multiple-access fading channels. They have shown that the boundary surface points are, in general, achieved by superposition coding and successive decoding techniques, and obtaining each boundary point can be associated with an optimization problem in which a weighted sum rate is maximized. Vishwanath et al. [6] derived the explicit optimal power and rate allocation schemes (similar to waterfilling) by considering that the users are successively decoded in the same order for all channel states. For the convex capacity region, the unique decoding order was shown to be the reverse order of the priority weight. While superposition coding and successive decoding strategies provide superior performance, time-division multiple access (TDMA) may in certain cases be preferred due to its simplicity. Note that the performance of TDMA approaches that of the optimal strategy as the signal-to-noise ratio (SNR) vanishes but, as shown by Caire et al. [7], TDMA is strictly suboptimal when SNR is low but nonzero.

While establishing the fundamental performance limits, the aforementioned studies have not explicitly taken into account buffer constraints and random arrivals. In [8] and [9], Yeh and Cohen considered multiaccess fading channels with random packet arrivals to buffered transmitters and characterized rate and power allocation strategies that maximize the stable throughput of the system. The maximum stable throughput region was shown in [10] to be the same as the MAC capacity region. In [11], the same authors investigated rate allocation policies that minimize the average packet delay in multiaccess fading channels again under the assumption of randomly arriving packets. More recently, Ehsan and Javidi [12] have studied delay optimal rate allocation strategies as well in two-user multiaccess channels but in the presence of asymmetric arrival processes, processing rates, and packet length distributions. Yang and Ulukus [13] also considered an asymmetric setting and analyzed how to control the transmission probabilities in order to minimize the average delay in a two-user multiaccess scenario.

In this paper, we also investigate the performance under buffer constraints but provide a perspective different from those of previous studies. In particular, we consider statistical quality of service $(\mathrm{QoS})$ constraints in the form of limitations on the buffer violation probabilities and study the achievable rate region under such constraints in multiaccess fading channels. Note that in certain delay sensitive applications, such as interactive or streaming video, constraints on delay bound violation probability may be required rather than limitations on the average delay. For this analysis, we employ the concept of effective capacity [14], which can be seen as the maximum constant arrival rate that a given time-varying service process 


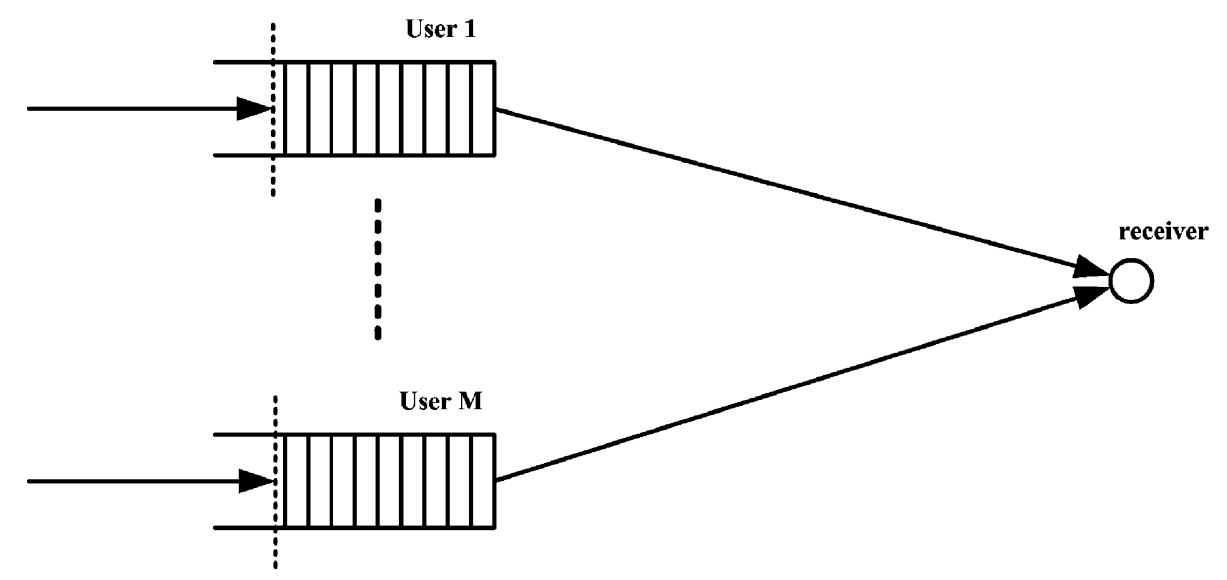

Fig. 1. System model.

can support while satisfying statistical QoS guarantees. The effective capacity formulation uses large deviations theory and incorporates the statistical QoS constraints by capturing the rate of decay of the buffer occupancy probability for large queue lengths. The analysis and application of effective capacity in various settings has attracted much interest recently (see e.g., [15]-[21] and references therein). In [18], Liu et al. considered a two-user cooperative multiple-access fading channel and analyzed the rate region achieved with frequency-division multiplexing when the users are operating under QoS constraints in the form of limitations on buffer overflow probabilities. In this study, cooperation among the users is shown to significantly improve the achievable rate region if the quality of the wireless link between the users is better than those of the links between the users and the destination. We note that since the transmitters are assumed to not know the channel conditions, power and rate adaptation policies are not studied in [18]. Additionally, since orthogonal transmission schemes are considered, superposition coding and successive decoding strategies are not addressed in detail.

Our contributions and major findings in this paper can be summarized as follows. We consider the scenario in which both the transmitters and the receiver have perfect channel side information (CSI). First, assuming that no power control is employed in the transmission, we characterize the rate regions for both superposition transmission strategies and TDMA. Unlike the results obtained in [1] and [6], varying the decoding order with respect to the channel states is shown to significantly increase the achievable rate region (i.e., throughput region) under QoS constraints. Also, it is demonstrated that time-sharing strategies among the vertex of the rate regions can no longer achieve the boundary surface of the throughput region. Additionally, we show that if we take the sum-rate throughput, or the sum effective capacity, as the performance metric, TDMA can, in certain cases, even achieve better performance than superposition coding when a fixed decoding order is employed at the receiver. Next, we incorporate power control policies into the model. For this case, we first obtain closed-form expressions for the optimal power control policies under the assumption that the decoding order is fixed at the receiver side. When the decoding order is variable, we identify the conditions the optimal power control policies should satisfy. We also describe an algorithm to determine such policies.

The remainder of this paper is organized as follows. Section II describes the system model. In Section III, effective capacity as a measure of the performance under statistical QoS constraints is briefly discussed, and the throughput region under QoS constraints is defined. In Section IV, under the assumption of no power control, we analyze the throughput region for both fixed and variable decoding order strategies. Section V describes the optimal power control policies. Finally, Section VI concludes this paper.

\section{System Model AND MAC CAPACITY REGION}

As shown in Fig. 1, we consider an uplink scenario where $M$ users with individual power and buffer constraints (i.e., QoS constraints) communicate with a single receiver. It is assumed that the transmitters generate data sequences which are divided into frames of duration $T$. These data frames are initially stored in the buffers before they are transmitted over the wireless channel. The discrete-time signal at the receiver in the $i$ th symbol duration is given by

$$
Y[i]=\sum_{j=1}^{M} h_{j}[i] X_{j}[i]+n[i], \quad i=1,2, \ldots
$$

where $M$ is the number of users, $X_{j}[i]$ and $h_{j}[i]$ denote the complex-valued channel input and the fading coefficient of the $j$ th user, respectively. We assume that $\left\{h_{j}[i]\right\}$ 's are jointly stationary and ergodic discrete-time processes, and we denote the magnitude-square of the fading coefficients by $z_{j}[i]=\left|h_{j}[i]\right|^{2}$. Above, $n[i]$ is a zero-mean, circularly symmetric, complex Gaussian random variable with variance $\mathbb{E}\left\{|n[i]|^{2}\right\}=N_{0}$. The additive Gaussian noise samples $\{n[i]\}$ are assumed to form an independent and identically distributed (i.i.d.) sequence. Finally, $Y[i]$ denotes the received signal.

The channel input of user $j$ is subject to an average energy constraint $\mathbb{E}\left\{\left|x_{j}[i]\right|^{2}\right\} \leq \bar{P}_{j} / B$ for all $j$, where $B$ is the bandwidth available in the system. Assuming that the symbol rate is $B$ complex symbols per second, we can see that this formulation indicates that user $j$ is subject to an average power constraint of $\bar{P}_{j}$. With these definitions, the average transmitted 
SNR of user $j$ is $\operatorname{SNR}_{j}=\frac{\bar{P}_{j}}{N_{0} B}$. Now, if we denote $P_{j}[\mathbf{z}]$ as the instantaneous transmit power as a function of the fading states $\mathbf{z}=\left(z_{1}, \ldots, z_{M}\right)$, the instantaneous transmitted SNR level becomes $\mu_{j}[\mathbf{z}]=\frac{P_{j}[\mathbf{z}]}{N_{0} B}$. Then, the average power constraint is equivalent to the average SNR constraint $\mathbb{E}\left\{\mu_{j}[\mathbf{z}]\right\} \leq \operatorname{SNR}_{j}$ for user $j$.

\section{A. Fixed Power and Variable Rate}

First, we consider the case in which the transmitters operate at fixed power and hence do not employ any power adaptation policies. The capacity region of this channel is given by [1], [3]

$$
\begin{aligned}
\mathcal{R}_{\mathrm{MAC}}=\{ & \left(R_{\mathrm{avg}, 1}, \ldots, R_{\mathrm{avg}, M}\right): \\
& \mathbf{R}_{\mathrm{avg}}(S) \leq B \mathbb{E}_{\mathbf{z}}\left\{\log _{2}\left(1+\sum_{j \in S} \mathrm{SNR}_{j} z_{j}\right)\right\} \\
& \forall S \subset\{1, \ldots, M\}\}
\end{aligned}
$$

where $\mathbf{R}_{\mathrm{avg}}(S)=\sum_{i \in S} R_{\mathrm{avg}, i}$. As well known, there are $M$ ! vertices of the polyhedron defined in (2). The vertex $\mathbf{R}_{\mathrm{avg}, \pi}=$ $\left(R_{\mathrm{avg}, \pi(1)}, \ldots, R_{\mathrm{avg}, \pi(M)}\right)$ corresponds to a permutation $\pi$, or the successive decoding order at the receiver, i.e., users are decoded in the order given by $\pi(1), \ldots, \pi(M)$. This vertex is specified by the average rates

$$
R_{\mathrm{avg}, \pi(k)}=B \mathbb{E}_{\mathbf{z}}\left\{\log _{2}\left(1+\frac{\mathrm{SNR}_{\pi(k)} z_{\pi(k)}}{1+\sum_{i=k+1}^{M} \mathrm{SNR}_{\pi(i)} z_{\pi(i)}}\right)\right\}
$$

in bits/s for $k=1, \ldots, M$. With this characterization, we see that for the given decoding order $\pi$, the maximum instantaneous service rate for user $\pi(k)$ is

$$
R_{\pi(k)}=B \log _{2}\left(1+\frac{\mathrm{SNR}_{\pi(k)} z_{\pi(k)}}{1+\sum_{i=k+1}^{M} \mathrm{SNR}_{\pi(i)} z_{\pi(i)}}\right) \text { bits/s. }
$$

Time sharing among these $M$ ! permutations of decoding orders yields any point on the boundary surface of $\mathcal{R}_{\mathrm{MAC}}$ [28]. As also discussed in [6], it can be easily verified that varying the decoding order according to the channel states does not provide any improvement on the capacity region.

\section{B. Variable Power and Variable Rate}

Now, we suppose that dynamic power and rate allocation is performed according to time variations in the channels. For a given set of power allocation policies $\mathcal{U}=\left\{\mu_{1}, \ldots, \mu_{M}\right\}$, where $\mu_{j} \geq 0$ is the power control policy of the $j$ th user, the achievable rate region is described by [3]

$$
\begin{gathered}
\mathcal{R}(\mathcal{U})=\left\{\mathbf{R}_{\text {avg }}: \mathbf{R}_{\text {avg }}(S) \leq \mathbb{E}_{\mathbf{z}}\left\{B \log _{2}\left(1+\sum_{j \in S} \mu_{j}(\mathbf{z}) z_{j}\right)\right\}\right. \\
\forall S \subset\{1, \ldots, M\}\} .
\end{gathered}
$$

For a given decoding order at the receiver, the individual average and instantaneous rates of the users can be obtained similar to (3) and (4), respectively, with SNR replaced by $\mu$. The capacity region is given by

$$
\mathcal{R}_{\mathrm{MAC}}=\bigcup_{\mathcal{U} \in \mathcal{F}} \mathcal{R}(\mathcal{U})
$$

where $\mathcal{F}$ is the set of all feasible power control policies that satisfy the average power constraint

$$
\mathcal{F} \equiv\left\{\mathcal{U}: \mathbb{E}_{\mathbf{z}}\left\{\mu_{j}(\mathbf{z})\right\} \leq \mathrm{SNR}_{j}, \mu_{j} \geq 0, \quad \forall j\right\} .
$$

\section{TDMA}

For simplicity, we assume that the time division strategy is fixed prior to transmission. Let $\delta_{j}$ denote the fraction of time allocated to user $j$. Note that we have $\sum_{j=1}^{M} \delta_{j}=1$. In each frame, each user occupies the entire bandwidth to transmit the signal in the corresponding fraction of time. Then, the instantaneous service rate for user $j$ in each frame is given by

$$
R_{j}\left(\mathrm{SNR}_{j}\right)=B \log _{2}\left(1+\frac{\mathrm{SNR}_{j}}{\delta_{j}} z_{j}\right) \text { bits } / \mathrm{s} .
$$

Note that user $j$ is assumed to transmit with the higher average power of $\bar{P}_{j} / \delta_{j}$ in the allocated $\delta_{j}$ fraction of the time.

\section{PRELIMINARIES}

\section{A. Effective Capacity}

In [14], Wu and Negi defined the effective capacity as the maximum constant arrival rate that a given service process can support in order to guarantee a statistical QoS requirement specified by the QoS exponent $\theta$. The effective capacity is formulated as

$$
C(\theta)=-\lim _{t \rightarrow \infty} \frac{1}{\theta t} \log _{e} \mathbb{E}\left\{e^{-\theta S[t]}\right\} \quad \text { bits } / \mathrm{s}
$$

where the expectation is with respect to $S[t]=\sum_{i=1}^{t} s[i]$, which is the time-accumulated service process, and $\{s[i], i=$ $1,2, \ldots\}$ denotes the discrete-time stationary and ergodic stochastic service process.

Operational meaning of the effective capacity is the following. If the constant arrival rate to the buffer is equal to the effective capacity $C(\theta)$, then the queue length process converges in distribution to a random variable $Q$ that satisfies [31]

$$
\lim _{q \rightarrow \infty} \frac{\log \operatorname{Pr}(Q \geq q)}{q}=-\theta .
$$

$Q$ can be seen as the stationary queue length, and $\theta$ as the asymptotic decay rate of the tail distribution of the queue length $Q$. Hence, effective capacity specifies the maximum constant arrival rate that can be supported by the time-varying channel, while the queue-overflow probability is guaranteed to behave as $\operatorname{Pr}(Q \geq q) \approx e^{-\theta q}$ for large overflow threshold $q$. Therefore, the QoS exponent $\theta$ can be regarded as a parameter that specifies the asymptotic exponential decay rate of the overflow probability and describes how strict the QoS constraints are. For instance, larger $\theta$ corresponds to more strict QoS constraints 
while smaller $\theta$ implies looser QoS guarantees. As noted in [16], when we have $\operatorname{Pr}(Q \geq q) \approx e^{-\theta q}$, then the delay violation probability can be approximated as $\operatorname{Pr}(D \geq d) \approx e^{-\theta \xi d}$ for large $d$, where $D$ denotes the steady-state delay experienced in the buffer and $\xi$ is determined by the arrival and service processes. In a more specific scenario in which the arrival rate is constant, Liu and Chamberland [19] showed that $\operatorname{Pr}(D>d) \leq$ $c \sqrt{\operatorname{Pr}(Q>q)}$ where $c$ is some constant, $q=a d$, and $a$ is the constant arrival rate.

Since the average arrival rate is equal to the average departure rate when the queue is in steady state [22], effective capacity, which characterizes the maximum constant arrival rate, can also be seen as the maximum throughput in the presence of constraints on the buffer or delay violation probabilities. Note that requiring the tail probabilities of buffer/delay violations to decay exponentially is a stronger condition than stability or having the average buffer length or delay to be finite. Therefore, throughput in the presence of QoS limitations will, in general, be less than the throughput under stability constraints.

In this paper, in order to simplify the analysis while considering general fading distributions, we assume that the fading coefficients stay constant over the frame duration $T$ and vary independently for each frame and each user. In this scenario, $s[i]=T R[i]$, where $R[i]$ is the instantaneous service rate in the $i$ th frame duration $[i T ;(i+1) T)$. Then, (9) can be written as

$$
C(\theta)=-\frac{1}{\theta T} \log _{e} \mathbb{E}_{\mathbf{z}}\left\{e^{-\theta T R[i]}\right\} \quad \operatorname{bits} / \mathrm{s}
$$

where $R[i]$ is, in general, a function of the fading state $\mathbf{z}$. Equation (11) is obtained using the fact that instantaneous rates $\{R[i]\}$ vary independently from one frame to another. It is interesting to note that as $\theta \rightarrow 0$ and hence QoS constraints relax, effective capacity approaches the ergodic capacity, i.e., $C(\theta) \rightarrow \mathbb{E}_{\mathbf{z}}\{R[i]\}$. On the other hand, as shown in [20], $C(\theta)$ converges to the delay limited capacity as $\theta$ grows without limit (i.e., $\theta \rightarrow \infty$ ) and QoS constraints become increasingly more strict. Therefore, effective capacity enables us to study the performance levels between the two extreme cases of delay limited capacity, which can be seen as a deterministic service guarantee or equivalently as a performance level attained under hard QoS limitations, and ergodic capacity, which is achieved in the absence of any QoS considerations.

Throughout the rest of the paper, we use the effective capacity normalized by bandwidth $B$, which is denoted by

$$
\mathrm{C}(\theta)=\frac{C(\theta)}{B} \quad \text { bits } / \mathrm{s} / \mathrm{Hz}
$$

At this point, it is also important to note that the transmission strategies (such as superposition coding schemes, TDMA methods, and power control policies) and reception strategies (such as the successive decoding order) will henceforth be designed and analyzed as functions of the fading states and the QoS exponent $\theta$. Hence, our transmission and reception policies take into account the statistical queueing constraints through the QoS exponents but not the actual queue lengths and states. We note that the authors in [25]-[27] have recently studied queuelength-based policies in the context of wireless scheduling in broadcast scenarios. In these works, only one user at a time is served by the transmitter in a downlink model. Shakkottai [25] investigated the effective capacity achieved by a greedy scheduling rule that picks the user with the highest channel rate and a max-queue rule that picks the user with the largest product of the queue length and the channel rate. Even though an i.i.d. channel model (akin to our block-fading assumption) is considered in this paper, it is described that the main difficulty in the analysis of queue-length-based policies arises from the fact that these policies statistically couple the rates allocated to various users across time. Therefore, due to correlation over time, the effective capacity formula in (9) cannot be simplified to that in (11). In such cases, effective capacity cannot be computed directly and certain technical difficulties are encountered. In particular, the techniques of sample path large deviations and calculus of variations are needed to determine the performance. In [25], these approaches are applied to relatively simple scenarios with two users, each of which experiences a two-state (ON-OFF) channel. More recently, using the sample-path large deviation principle, Venkataramanan and Lin [26] studied wireless scheduling algorithms that maximize asymptotic decay rate of the queue-overflow probability in a more general downlink scenario with $N$ users and $M$ possible channel states.

In this paper, we consider more complex channel models with continuous fading and more sophisticated transmission strategies such as superposition coding (rather than orthogonal transmissions) and power control techniques in a multiple-access scenario. By addressing only the statistical queueing constraints, we formulate a tractable problem for more practically appealing system models. At the same time, we note that queue-length-based policies have the potential to attain a higher effective capacity than those achieved by greedy policies that take into account only the channel states (see e.g., [25, Fig. 3]). Hence, from this perspective, our results, which incorporate the channel states and the QoS exponents but not the actual queue states in the transmission and reception, can be regarded as baselines with which the performances of queue-length-based policies can be compared.

\section{B. Throughput Region}

Suppose that $\Theta=\left(\theta_{1}, \ldots, \theta_{M}\right)$ is a vector composed of the QoS constraints of $M$ users. Let $\mathrm{C}(\Theta)=$ $\left(C_{1}\left(\theta_{1}\right), \ldots, C_{M}\left(\theta_{M}\right)\right)$ denote the vector of the normalized effective capacities. We first have the following characterization.

Definition 1: The effective throughput region is described as

$$
\begin{aligned}
& \mathcal{C}_{\mathrm{MAC}}(\Theta, \mathbf{S N R}) \\
& =\bigcup_{\mathbf{R}}\left\{\mathrm{C}(\Theta) \geq \mathbf{0}: \mathrm{C}_{j}\left(\theta_{j}\right) \leq-\frac{1}{\theta_{j} T B} \log _{e} \mathbb{E}_{\mathbf{z}}\left\{e^{-\theta T R_{j}}\right\}\right\} \\
& \text { s.t. } \mathbb{E}\{\mathbf{R}\} \in \mathcal{R}_{\mathrm{MAC}}
\end{aligned}
$$

where $\mathbf{R}=\left\{R_{1}, R_{2}, \ldots, R_{M}\right\}$ represents the vector composed of the instantaneous transmission (or equivalently service) rates of $M$ users. Note that the union is over the distributions of the vector $\mathbf{R}$ such that the expected value $\mathbb{E}\{\mathbf{R}\}$ lies in the MAC capacity region. 
Remark 1: The throughput region given in Definition 1 represents the set of all vectors of constant arrival rates $C(\theta)$ that can be supported in the fading MAC in the presence QoS constraints specified by $\Theta=\left(\theta_{1}, \ldots, \theta_{M}\right)$. Since reliable communication is considered, the arrival rates are supported by instantaneous service rates whose expected values are in the MAC capacity region. For instance, in the absence of power control, the maximum instantaneous service rates for a given decoding order are given by (4).

Using the convexity of the MAC capacity region $\mathcal{R}_{\mathrm{MAC}}$, we obtain the following preliminary result on the effective throughput region defined in (13).

Theorem 1: The throughput region $\mathcal{C}_{\mathrm{MAC}}(\Theta, \mathrm{SNR})$ is convex.

Proof: Let the vectors $\mathrm{C}(\Theta)$ and $\mathrm{C}^{\prime}(\Theta)$ belong to $\mathcal{C}_{\mathrm{MAC}}(\Theta$, SNR $)$. Then, there exist some rate vectors $\mathbf{R}$ and $\mathbf{R}^{\prime}$ for $C(\Theta)$ and $C^{\prime}(\Theta)$, respectively, such that $\mathbb{E}\{\mathbf{R}\}$ and $\mathbb{E}\left\{\mathbf{R}^{\prime}\right\}$ are in the MAC capacity region. By a time-sharing strategy, for any $\alpha \in(0,1)$, we know from the convexity of the MAC capacity region that $\mathbb{E}\left\{\alpha \mathbf{R}+(1-\alpha) \mathbf{R}^{\prime}\right\} \in \mathcal{R}_{\mathrm{MAC}}$. Now, we can write

$$
\begin{aligned}
& \alpha \mathrm{C}(\Theta)+(1-\alpha) \mathrm{C}^{\prime}(\Theta) \\
& \leq-\frac{1}{\Theta T B} \log _{e}\left(\mathbb{E}\left\{e^{-\Theta T \mathbf{R}}\right\}\right)^{\alpha}\left(\mathbb{E}\left\{e^{-\Theta T \mathbf{R}^{\prime}}\right\}\right)^{1-\alpha} \\
& =-\frac{1}{\Theta T B} \log _{e}\left(\mathbb{E}\left\{\left(e^{-\Theta T \alpha \mathbf{R}}\right)^{\frac{1}{\alpha}}\right\}\right)^{\alpha} \\
& \quad \times\left(\mathbb{E}\left\{\left(e^{-\Theta T(1-\alpha) \mathbf{R}^{\prime}}\right)^{\frac{1}{1-\alpha}}\right\}\right)^{1-\alpha} \\
& \leq-\frac{1}{\Theta T B} \log _{e} \mathbb{E}\left\{e^{-\Theta T\left(\alpha \mathbf{R}+(1-\alpha) \mathbf{R}^{\prime}\right)}\right\} .
\end{aligned}
$$

In (14)-(16), all operations, including the logarithm and exponential functions and expectations, are component-wise operations. For instance, the expression in (14) denotes a vector whose components are $\left\{\frac{1}{\theta_{j} T B} \log _{e}\left(\mathbb{E}\left\{e^{-\theta_{j} T R_{j}}\right\}\right)^{\alpha}\left(\mathbb{E}\left\{e^{-\theta T R_{j}^{\prime}}\right\}\right)^{1-\alpha}\right\}_{j=1}^{M}$. Similarly, the inequalities in (14) and (16) are component-wise inequalities. The inequality in (14) follows from the definition in (13). Moreover, (16) follows from Hölder's inequality and leads to the conclusion that $\alpha \mathrm{C}+(1-\alpha) \mathrm{C}^{\prime}$ still lies in the throughput region, proving the convexity result.

We are interested in the boundary of the region $\mathcal{C}_{\text {MAC }}(\Theta$, SNR $)$. Now that $\mathcal{C}_{\text {MAC }}(\Theta$, SNR $)$ is convex, we can characterize the boundary surface by considering the following optimization problem [3]:

$$
\max \lambda \cdot \mathrm{C}(\Theta) \text { subject to: } \mathrm{C}(\Theta) \in \mathcal{C}_{\mathrm{MAC}}(\Theta, \text { sNR }) .
$$

For all priority vectors, $\lambda=\left(\lambda_{1}, \ldots, \lambda_{M}\right)$ in $\mathfrak{R}_{+}^{M}$ with $\sum_{j=1}^{M} \lambda_{j}=1$.

\section{TRAnsmissions Without POWER CONTROL}

In this section, we assume that the signals are transmitted at a constant power level in each frame and hence power adaptation with respect to the fading states is not performed. Under this assumption, we initially consider the scenario in which the receiver decodes the users in a fixed order. Subsequently, we analyze the case of variable decoding order.

\section{A. Fixed Decoding Order}

We first assume that the receiver decodes the users in a fixed order in each frame. Hence, the decoding order does not change with respect to the realizations of the fading coefficients. If a single decoding order is used in the frame, it is obvious that only the vertices of the boundary region can be achieved. We consider a slightly more general case in which time-sharing technique is employed in each frame among different decoding orders. Note that the time-sharing strategy is also independent of the channel states and hence is fixed in different blocks. We denote the fraction of time allocated to decoding order $\pi_{m}$ as $\tau_{m}$. Naturally, the fractions of time satisfy $\tau_{m} \geq 0$ and $\sum_{m=1}^{M !} \tau_{m}=1$. Varying the values of $\tau_{m}$ enables us to characterize the throughput region. Under these assumptions, the effective capacity for each user on the boundary surface is

$$
C_{j}\left(\theta_{j}\right)=-\frac{1}{\theta_{j} T B} \log _{e} \mathbb{E}_{\mathbf{z}}\left\{e^{-\theta_{j} T \sum_{m=1}^{M !} \tau_{m} R_{\pi_{m}^{-1}(j)}}\right\}
$$

where $R_{\pi_{m}^{-1}(j)}$ represents the maximal instantaneous service rate of user $j$ at a given decoding order $\pi_{m}$, which is given by

$$
R_{\pi_{m}^{-1}(j)}=B \log _{2}\left(1+\frac{\mathrm{SNR}_{j} z_{j}}{1+\sum_{\pi_{m}^{-1}(i)>\pi_{m}^{-1}(j)} \mathrm{SNR}_{i} z_{i}}\right)
$$

where $\pi_{m}^{-1}$ is the inverse trace function of $\pi_{m}$.

Remark 2: Note that $R_{\pi_{m}^{-1}(j)}$ is the maximum instantaneous service rate achieved with superposition coding and a particular decoding order. Hence, the corresponding effective capacities characterize the throughput achieved with this strategy in the presence of QoS constraints. Note also that $R_{\pi_{m}^{-1}(j)}$, which represents the information-theoretic limit for instantaneous rates, can be approached if codes with large blocklengths are employed. Therefore, in order to have operational significance in the results, we assume throughout the paper that the number of symbols $T B$ in a frame duration of $T$ seconds is sufficiently large. If $T B$ is relatively small, rates attained with finite blocklength channel codes in the presence of possible decoding errors should be considered as addressed in [23] and [24].

Remark 3: Throughout the rest of the paper, we generally specify the effective capacity values on the boundary surface for simplicity and brevity. Effective capacity regions can immediately be specified using these boundary points. For instance, the effective capacity (or equivalently throughput) region for superposition coding and fixed decoding order is

$$
\begin{aligned}
\bigcup_{\left\{\tau_{m}\right\}}\{C(\Theta) & \geq \mathbf{0}: \\
C_{j}\left(\theta_{j}\right) & \left.\leq-\frac{1}{\theta_{j} T B} \log _{e} \mathbb{E}_{\mathbf{z}}\left\{e^{-\theta_{j} T \sum_{m=1}^{M !} \tau_{m} R_{\pi_{m}^{-1}(j)}}\right\}\right\}
\end{aligned}
$$

where the union is over different time allocation strategies.

Next, for comparison, we consider the TDMA case in which we also have similar time allocation strategies but only one user 
transmits in its specific fraction of time. We first have the following definition.

Definition 2: The throughput region for TDMA can be seen as the achievable vectors of arrival rates with each component bounded by the effective capacity obtained when the instantaneous service rate is given by (8). More specifically, the maximum effective capacity for user $j$ is

$$
\mathrm{C}_{j}^{\mathrm{TD}}\left(\theta_{j}\right)=-\frac{1}{\theta_{j} T B} \log _{e} \mathbb{E}\left\{e^{-\delta_{j} \theta_{j} T B \log _{2}\left(1+\frac{\mathrm{SNR}_{j}}{\delta_{j}} z_{j}\right)}\right\}
$$

where $\delta_{j}$ is the fraction of time allocated to user $j$, and $0 \leq \delta_{j} \leq$ 1 . We again assume that $\delta_{j} T B$ is sufficiently large so that the expression in (8) is a realistic representation of the service rate.

An immediate result can be obtained as follows.

Theorem 2: The throughput region for TDMA is convex.

Proof: Note that the points on the boundary surface is given in (21). Consider the function $f(\delta)=$ $-\delta \theta T B \log _{2}\left(1+\frac{\mathrm{SNR}}{\delta} z\right)$. It can be easily verified that $f(\delta)$ is a convex function in $\delta$. Then, $e^{f(\delta)}$ is a log-convex function. Since weighted nonnegative sum preserves the log-convexity [29, Sec. 3.5], we know that $\mathbb{E}_{z}\left\{e^{f(\delta)}\right\}$ is log-convex. Then, $-\frac{1}{\theta T B} \log _{e} \mathbb{E}\left\{e^{-\delta \theta T B \log _{2}\left(1+\frac{\mathrm{SNR}}{\delta} z\right)}\right\}$ is a concave function in $\delta$. Hence, we immediately see that the throughput region for TDMA is convex.

The optimal time allocation policy that maximizes the weighted sum can be obtained through the optimization problem

$$
\begin{gathered}
\max _{\left\{\delta_{j}\right\}} \sum_{j=1}^{M}-\frac{\lambda_{j}}{\theta_{j} T B} \log _{e} \mathbb{E}\left\{e^{-\delta_{j} \theta_{j} T B \log _{2}\left(1+\frac{\mathrm{SNR}_{j}}{\delta_{j}} z_{j}\right)}\right\}, \\
\text { s.t. } \sum_{j=1}^{M} \delta_{j}=1, \delta_{j} \geq 0 .
\end{gathered}
$$

The objective function in the above problem is concave, and we can use the Lagrangian maximization approach. Taking the derivative of the Lagrangian function with respect to $\delta_{j}$, we obtain, for each user, the optimality condition given in (23), shown at the bottom of the page, where $\kappa$ is the Lagrange multiplier whose value is chosen to satisfy the constraint $\sum_{j=1}^{M} \delta_{j}=1$. If the optimal value of $\delta_{j}$ turns out to be negative, then the optimal value of $\delta_{j}$ should be 0 . When $\lambda_{1}=\lambda_{2}=\cdots=\lambda_{M}$, the obtained values of $\left\{\delta_{j}\right\}$ are the ones that achieve the maximal sum-rate throughput, i.e., the sum of the effective capacities of the users. Although obtaining closed-form solutions is unlikely, the maximization problem in (22) can be easily solved numerically using convex optimization tools. Numerical results are provided in Section IV-C.

\section{B. Variable Decoding Order}

We now study the case in which the receiver varies the decoding order with respect to the fading states $\mathbf{z}=\left(z_{1}, \ldots, z_{M}\right)$. In its most general form, we assume that the receiver, for each fading state $\mathbf{z}$, employs a time sharing of the decoding orders in which the fraction of time allocated to decoding order $\pi_{m}$ is $\tau_{m}(\mathbf{z})$ for $m=1, \ldots M !$. Hence, for each fading state $\mathbf{z}$, the receiver now has the freedom to use possibly a different decoding order or a different time sharing of multiple decoding orders. For a given time-sharing policy $\left\{\tau_{m}(\mathbf{z})\right\}_{m=1}^{M !}$, the effective capacity of user $j$ is

$$
C_{j}\left(\theta_{j}\right)=-\frac{1}{\theta_{j} T B} \log _{e} \mathbb{E}_{\mathbf{z}}\left\{e^{-\theta_{j} T \sum_{m=1}^{M !} \tau_{m}(\mathbf{z}) R_{\pi_{m}^{-1}(j)}}\right\}
$$

where $R_{\pi_{m}^{-1}(j)}$ is given by (19). In this scheme, the instantaneous transmission rates for the users are selected from any point on the dominant face of the MAC instantaneous capacity region.

A more restrictive but simpler scheme for the receiver is to eliminate the time sharing and employ a particular single decoding order for each fading state $\mathbf{z}$. In this case, the instantaneous transmission rates are chosen from the vertices of the MAC instantaneous capacity region. More specifically, we assume that the vector space $\mathfrak{R}_{+}^{M}$ of the possible values for $\mathbf{z}$ is partitioned into $M$ ! disjoint regions $\left\{\mathcal{Z}_{m}\right\}_{m=1}^{M !}$ with respect to decoding orders $\left\{\pi_{m}\right\}_{m=1}^{M !}$. Hence, each region corresponds to a unique decoding order. For instance, when $\mathbf{z} \in \mathcal{Z}_{1}$, the receiver decodes the information in the order $\pi_{1}$. Therefore, this scheme corresponds to the special case of the general time-sharing approach with $\tau_{m}(\mathbf{z})=1$ when $\mathbf{z} \in \mathcal{Z}_{m}$ and zero otherwise for all $m=1, \ldots M$ !.

Now, for a given partition $\left\{\mathcal{Z}_{m}\right\}_{m=1}^{M !}$, the maximum effective capacity that can be achieved by the $j$ th user is

$$
\begin{aligned}
C_{j}\left(\theta_{j}\right) & =-\frac{1}{\theta_{j} T B} \log _{e} \mathbb{E}_{\mathbf{z}}\left\{e^{-\theta_{j} T R_{j}}\right\} \\
& =-\frac{1}{\theta_{j} T B} \log _{e}\left(\sum_{m=1}^{M !} \int_{\mathbf{z} \in \mathcal{Z}_{m}} e^{-\theta_{j} T R_{\pi_{m}^{-1}(j)}} p_{\mathbf{z}}(\mathbf{z}) d \mathbf{z}\right)
\end{aligned}
$$

$$
\frac{\partial \mathcal{J}}{\partial \delta_{j}}=\lambda_{j} \frac{\left.\mathbb{E}\left\{e^{-\delta_{j} \theta_{j} T B \log _{2}\left(1+\frac{\mathrm{SNR}_{j}}{\delta_{j}} z_{j}\right.}\right)\left(\log _{2}\left(1+\frac{\mathrm{SNR}_{j}}{\delta_{j}} z_{j}\right)-\frac{\frac{\mathrm{SNR}_{j}}{\delta_{j}} z_{j}}{1+\frac{\mathrm{SNR}_{j}}{\delta_{j}} z_{j}} \log _{2} e\right)\right\}}{\mathbb{E}\left\{e^{-\delta_{j} \theta_{j} T B \log _{2}\left(1+\frac{\mathrm{SNR}_{j}}{\delta_{j}} z_{j}\right)}\right\}}-\kappa=0
$$


where $p_{\mathbf{z}}$ is the distribution function of $\mathbf{z}$ and $R_{\pi_{m}^{-1}(j)}$ is given in (19). Akin to the optimization in (17), the optimal partition $\left\{\mathcal{Z}_{m}\right\}_{m=1}^{M !}$ that maximizes the weighted sum of the effective capacities can be identified by solving the following optimization problem:

$$
\begin{aligned}
\max _{\left\{\mathcal{Z}_{m}\right\}} \lambda \cdot \mathrm{C}(\Theta) & =\max _{\left\{\mathcal{Z}_{m}\right\}} \sum_{j=1}^{M} \lambda_{j} \mathrm{C}_{j}\left(\theta_{j}\right) \\
= & \max _{\left\{\mathcal{Z}_{m}\right\}} \sum_{j=1}^{M}-\frac{\lambda_{j}}{\theta_{j} T B} \\
& \times \log _{e}\left(\sum_{m=1}^{M !} \int_{\mathbf{z} \in \mathcal{Z}_{m}} e^{-\theta_{j} T R_{\pi_{m}^{-1}(j)}} p_{\mathbf{z}}(\mathbf{z}) d \mathbf{z}\right) .
\end{aligned}
$$

Note that the optimal partition depends on the weight vector $\lambda=\left(\lambda_{1}, \ldots, \lambda_{M}\right)$. By solving a sequence of optimization problems for different values of $\lambda$, we can trace the boundary of the effective throughput region.

Considering the expression for effective capacity and the optimization problem in (28), we note that finding closed-form analytical expressions for the optimal partitions of the channel state space seems intractable for a general scenario. With this in mind, we consider a simplified case in which all users have the same QoS constraint described by $\theta$. This case arises, for instance, if users do not have priorities over others in terms of buffer limitations or delay constraints.

1) Two-User MAC: First, we consider the two-user MAC case and suppose that the two users have the same QoS exponent $\theta$. Similar to the discussion in [21], finding an optimal decodingorder function can be reduced to finding a function $z_{2}=g\left(z_{1}\right)$ in the state space such that users are decoded in the order $(1,2)$ if $z_{2}<g\left(z_{1}\right)$ and users are decoded in the order $(2,1)$ if $z_{2}>$ $g\left(z_{1}\right)$. Hence, the function $g$ partitions the space of the possible values of $\mathbf{z}=\left(z_{1}, z_{2}\right)$. With this, the optimization problem in (27) becomes

$$
\max _{g} \lambda_{1} C_{1}\left(\theta, g\left(z_{1}\right)\right)+\left(1-\lambda_{1}\right) C_{2}\left(\theta, g\left(z_{1}\right)\right)
$$

where $\mathrm{C}_{1}\left(\theta, g\left(z_{1}\right)\right)$ and $\mathrm{C}_{2}\left(\theta, g\left(z_{1}\right)\right)$ are expressed as

$$
\begin{aligned}
& \mathrm{C}_{1}\left(\theta, g\left(z_{1}\right)\right) \\
& =\frac{-1}{\theta T B} \log _{e}\left(\int_{0}^{\infty} \int_{g\left(z_{1}\right)}^{\infty} e^{-\theta T B \log _{2}\left(1+\mathrm{SNR}_{1} z_{1}\right)} p_{\mathbf{z}}\left(z_{1}, z_{2}\right) d z_{2} d z_{1}\right. \\
& \quad+\int_{0}^{\infty} \int_{0}^{g\left(z_{1}\right)_{-}-\theta T B \log _{2}\left(1+\frac{\mathrm{SNR}_{1} z_{1}}{1+\mathrm{SNR}_{2} z_{2}}\right)} p_{\left.p_{\mathbf{z}}\left(z_{1}, z_{2}\right) d z_{2} d z_{1}\right)}
\end{aligned}
$$$$
\mathrm{C}_{2}\left(\theta, g\left(z_{1}\right)\right)
$$$$
=\frac{-1}{\theta T B} \log _{e}\left(\int_{0}^{\infty} \int_{0}^{g\left(z_{1}\right)} e^{-\theta T B \log _{2}\left(1+\mathrm{SNR}_{2} z_{2}\right)} p_{\mathbf{z}}\left(z_{1}, z_{2}\right) d z_{2} d z_{1}\right.
$$$$
\left.+\int_{0}^{\infty} \int_{g\left(z_{1}\right)}^{\infty} e^{-\theta T B \log _{2}\left(1+\frac{\mathrm{SNR}_{2} z_{2}}{1+\mathrm{SNR}_{1} z_{1}}\right)} p_{\mathbf{z}}\left(z_{1}, z_{2}\right) d z_{2} d z_{1}\right) .
$$

Note that the maximization in (29) is over the choice of the function $g\left(z_{1}\right)$. Implicitly, $g\left(z_{1}\right)$ should always be larger than zero as implied in (30) and (31). In cases in which this condition is not satisfied, we need to find a function $z_{1}=f\left(z_{2}\right)$ instead, as will be specified below.

Theorem 3: The optimal decoding order as a function of the fading state $\mathbf{z}=\left(z_{1}, z_{2}\right)$ for a specific common QoS constraint $\theta$ in the two-user case is characterized by the following functions:

$$
\begin{aligned}
& g\left(z_{1}\right)=\frac{\left(1+\mathrm{SNR}_{1} z_{1}\right) K^{\frac{1}{\beta}}-1}{\mathrm{SNR}_{2}}, \quad \text { if } K \in[1, \infty) \\
& f\left(z_{2}\right)=\frac{\left(1+\mathrm{SNR}_{2} z_{2}\right) K^{-\frac{1}{\beta}}-1}{\mathrm{SNR}_{1}}, \quad \text { if } K \in[0,1)
\end{aligned}
$$

where $\beta=\frac{\theta T B}{\log _{e} 2}$ and $K \in[0, \infty)$ is a constant that depends on the weight $\lambda_{1}$ in (29) and the values of the double integrals in (30) and (31). Note that the function used to partition the state space is either $g$ or $f$ depending on the value of $K$.

Proof: Suppose that the optimal decoding order is specified by the function $z_{2}=g\left(z_{1}\right)$. We define

$$
\mathcal{J}\left(\hat{g}\left(z_{1}\right)\right)=\lambda_{1} \mathrm{C}_{1}\left(\theta, \hat{g}\left(z_{1}\right)\right)+\left(1-\lambda_{1}\right) \mathrm{C}_{2}\left(\theta, \hat{g}\left(z_{1}\right)\right)
$$

where $\hat{g}\left(z_{1}\right)=g\left(z_{1}\right)+s \eta\left(z_{1}\right) \cdot g\left(z_{1}\right)$ is the optimal function, $s$ is any constant, and $\eta\left(z_{1}\right)$ represents arbitrary perturbation. A necessary condition that needs to be satisfied is [30]

$$
\left.\frac{d}{d s}\left(\mathcal{J}\left(\hat{g}\left(z_{1}\right)\right)\right)\right|_{s=0}=0 .
$$

We define the following:

$$
\begin{aligned}
\phi_{1}= & \int_{0}^{\infty} \int_{g\left(z_{1}\right)}^{\infty} e^{-\theta T B \log _{2}\left(1+\mathrm{SNR}_{1} z_{1}\right)} p_{\mathbf{z}}\left(z_{1}, z_{2}\right) d z_{2} d z_{1} \\
& +\int_{0}^{\infty} \int_{0}^{g\left(z_{1}\right)} e^{-\theta T B \log _{2}\left(1+\frac{\mathrm{SNR}_{1} z_{1}}{1+\mathrm{SNR}_{2} z_{2}}\right)} p_{\mathbf{z}}\left(z_{1}, z_{2}\right) d z_{2} d z_{1} \\
\phi_{2}= & \int_{0}^{\infty} \int_{0}^{g\left(z_{1}\right)} e^{-\theta T B \log _{2}\left(1+\mathrm{SNR}_{2} z_{2}\right)} p_{\mathbf{z}}\left(z_{1}, z_{2}\right) d z_{2} d z_{1} \\
& +\int_{0}^{\infty} \int_{g\left(z_{1}\right)}^{\infty} e^{-\theta T B \log _{2}\left(1+\frac{\mathrm{SNR}_{2} z_{2}}{1+\mathrm{SNR}_{1} z_{1}}\right)_{p_{\mathbf{z}}\left(z_{1}, z_{2}\right) d z_{2} d z_{1}}}
\end{aligned}
$$

By noting that $\frac{d \hat{g}\left(z_{1}\right)}{d s}=\eta\left(z_{1}\right)$, and from (35)-(37), we can derive

$$
\begin{aligned}
\int_{0}^{\infty} & \left(-\frac{\lambda_{1}}{\theta T B \phi_{1}}\left(\left(1+\frac{\mathrm{SNR}_{1} z_{1}}{1+\mathrm{SNR}_{2} g\left(z_{1}\right)}\right)^{-\beta}-\left(1+\mathrm{SNR}_{1} z_{1}\right)^{-\beta}\right)\right. \\
- & \left.\frac{1-\lambda_{1}}{\theta T B \phi_{2}}\left(\left(1+\mathrm{SNR}_{2} g\left(z_{1}\right)\right)^{-\beta}-\left(1+\frac{\mathrm{SNR}_{2} g\left(z_{1}\right)}{1+\mathrm{SNR}_{1} z_{1}}\right)^{-\beta}\right)\right) \\
\cdot & p_{\mathbf{z}}\left(z_{1}, g\left(z_{1}\right)\right) \eta\left(z_{1}\right) d z_{1}=0 .
\end{aligned}
$$

Since the aforementioned equation holds for any $\eta\left(z_{1}\right)$, it follows that

$$
-\frac{\lambda_{1}}{\theta T B \phi_{1}}\left(\left(1+\frac{\mathrm{SNR}_{1} z_{1}}{1+\mathrm{SNR}_{2} g\left(z_{1}\right)}\right)^{-\beta}-\left(1+\mathrm{SNR}_{1} z_{1}\right)^{-\beta}\right)
$$




$$
-\frac{1-\lambda_{1}}{\theta T B \phi_{2}}\left(\left(1+\mathrm{SNR}_{2} g\left(z_{1}\right)\right)^{-\beta}-\left(1+\frac{\mathrm{SNR}_{2} g\left(z_{1}\right)}{1+\mathrm{SNR}_{1} z_{1}}\right)^{-\beta}\right)=0
$$

which after rearranging and defining $K$ as follows yields

$$
\frac{\left(1+\frac{\mathrm{SNR}_{1} z_{1}}{1+\mathrm{SNR}_{2} g\left(z_{1}\right)}\right)^{-\beta}-\left(1+\mathrm{SNR}_{1} z_{1}\right)^{-\beta}}{\left(1+\frac{\mathrm{SNR}_{2} g\left(z_{1}\right)}{1+\mathrm{SNR}_{1} z_{1}}\right)^{-\beta}-\left(1+\mathrm{SNR}_{2} g\left(z_{1}\right)\right)^{-\beta}}=\frac{\left(1-\lambda_{1}\right) \phi_{1}}{\lambda_{1} \phi_{2}}=K \text {. }
$$

Obviously, $K \geq 0$. Notice that after a simple computation, (40) becomes

$$
\left(\frac{1+\mathrm{SNR}_{1} z_{1}}{1+\mathrm{SNR}_{2} g\left(z_{1}\right)}\right)^{-\beta}=K
$$

which leads to (32) after rearranging. Note here that if $K<1$, $g\left(z_{1}\right)<0$ for $z_{1}<\frac{K^{-\frac{1}{\beta}}-1}{\operatorname{SNR}_{1}}$. Then, the expressions in (30) and (31) are not well defined. In this case, we denote the optimal function as $z_{1}=f\left(z_{2}\right)$ instead. Following a similar approach as shown in (30)-(41) yields (33).

Remark 4: We have assumed that the users are decoded in the order $(1,2)$ when $z_{2}<g\left(z_{1}\right)$ (or $z_{1}>f\left(z_{2}\right)$ if $K<1$ ) and decoded in the order $(2,1)$ when $z_{2}>g\left(z_{1}\right)$ (or $z_{1}<f\left(z_{2}\right)$ if $K<1$ ). It is interesting note that if we switch the decoding orders in the regions (i.e., if users are decoded in the order (1, 2) when $z_{2}>g\left(z_{1}\right)$ ), exactly the same partition functions as in (32) and (33) are obtained due to the symmetric nature of the problem. Hence, the structure of the optimal functions that partition the space of channel states $\left(z_{1}, z_{2}\right)$ into two nonoverlapping regions does not depend on which decoding order is used in which region.

Remark 5: Although the partition does not depend on the choice of the decoding orders in different regions, the performance definitely does. Our numerical computations show that the order selected originally at the beginning of our discussion (i.e., using the decoding order $(1,2)$ when $z_{2}<g\left(z_{1}\right)$ or $\left.z_{1}>f\left(z_{2}\right)\right)$ provides a larger throughput region than otherwise. This observation leads to an interesting conclusion. Note that partition functions $g\left(z_{1}\right)$ in (32) and $f\left(z_{2}\right)$ in (33) are linear functions of $z_{1}$ and $z_{2}$, respectively. When $K \geq 1$ and

$$
z_{2}<g\left(z_{1}\right)=\frac{\left(1+\mathrm{SNR}_{1} z_{1}\right) K^{\frac{1}{\beta}}-1}{\mathrm{SNR}_{2}}
$$

user 1 is decoded first and user 2 is decoded last. Hence, for instance, when $z_{1}$ is much larger than $z_{2}$ and user 1 is enjoying much better channel conditions, user 1 is decoded first in the presence of interference caused by user 2's received signal. User 2 , who has less favorable conditions, is decoded subsequently without experiencing any interference. Note that such an operation is the opposite of an opportunistic behavior and leads to a more fair treatment of users. This is rather insightful since the users are assumed to operate under similar QoS limitations (i.e., they have the same QoS exponent $\theta$ ). Note that if the decoding orders are switched, users having favorable channel conditions will be decoded last and hence experience no interference. In such a case, there is a bias toward users with better channel conditions, which leads to inefficient performance when both users operate under similar buffer constraints.

Our earlier observations have led us to propose the following suboptimal decoding-order strategy for a scenario with more than 2 users.

2) Suboptimal Decoding Order: In this section, we consider an arbitrary number of users. When all users have the same QoS constraint specified by $\theta$, we propose a suboptimal decoding order given by

$$
\frac{\lambda_{\pi(1)}}{z_{\pi(1)}} \leq \frac{\lambda_{\pi(2)}}{z_{\pi(2)}} \cdots \leq \frac{\lambda_{\pi(M)}}{z_{\pi(M)}}
$$

due to the observation that the user with the largest weight $\lambda$ should be decoded last, and the fact that the higher the value of $z$, the less power is needed to achieve a specific effective capacity. Considering a two-user example, we, with this choice of the decoding order, can express the points on the boundary surface as

$$
\begin{aligned}
& \mathrm{C}_{1}(\theta)= \\
& -\frac{1}{\theta T B} \log _{e}\left(\int_{0}^{\infty} \int_{\frac{\lambda_{2} z_{1}}{\lambda_{1}}}^{\infty} e^{-\theta T B \log _{2}\left(1+\mathrm{SNR}_{1} z_{1}\right)} p_{\mathbf{z}}\left(z_{1}, z_{2}\right) d z_{2} d z_{1}\right. \\
& \quad+\int_{0}^{\infty} \int_{0}^{\frac{\lambda_{2} z_{1}}{\lambda_{1}}-\theta T B \log _{2}\left(1+\frac{\mathrm{SNR}_{1} z_{1}}{1+\mathrm{SNR}_{2} z_{2}}\right)} e_{\left.\mathbf{z}_{\mathbf{z}}\left(z_{1}, z_{2}\right) d z_{2} d z_{1}\right)}
\end{aligned}
$$

$$
\begin{aligned}
& \mathrm{C}_{2}(\theta)= \\
& -\frac{1}{\theta T B} \log _{e}\left(\int_{0}^{\infty} \int_{0}^{\frac{\lambda_{2} z_{1}}{\lambda_{1}}} e^{-\theta T B \log _{2}\left(1+\mathrm{SNR}_{2} z_{2}\right)} p_{\mathbf{z}}\left(z_{1}, z_{2}\right) d z_{2} d z_{1}\right. \\
& \left.\quad+\int_{0}^{\infty} \int_{\frac{\lambda_{2} z_{1}}{\lambda_{1}}}^{\infty} e^{-\theta T B \log _{2}\left(1+\frac{\mathrm{SNR}_{2} z_{2}}{1+\mathrm{SNR}_{1} z_{1}}\right)} p_{\mathbf{z}}\left(z_{1}, z_{2}\right) d z_{2} d z_{1}\right)
\end{aligned}
$$

\section{Numerical Results}

We have performed numerical analysis for independent Rayleigh fading channels with $\mathbb{E}\{\mathbf{z}\}=1$. In Fig. 2, the throughput region of a two-user MAC is plotted for superposition strategies with different decoding ordering methods at the receiver, and also for TDMA. In the figure, the solid and dotted curves provide the throughput regions achieved by employing optimal and suboptimal variable decoding orders, respectively, at the receiver. Note that in the optimal strategy described by the results of Theorem 3, the receiver chooses the decoding order according to the channel states such that the weighted sum of effective capacities, i.e., summation of log-moment generating functions, is maximized. We see that the suboptimal strategy described in Section IV-B2 can achieve almost the same rate region as the optimal strategy, indicating the efficiency of this approach. In the same figure, dot-dashed curve provides the throughput region achieved by employing a fixed decoding order for all channel states. Here, we observe that the strategy of using a fixed decoding order at the receiver is strictly suboptimal even when the users are operating under similar buffer constraints, and varying the decoding order with the respect 


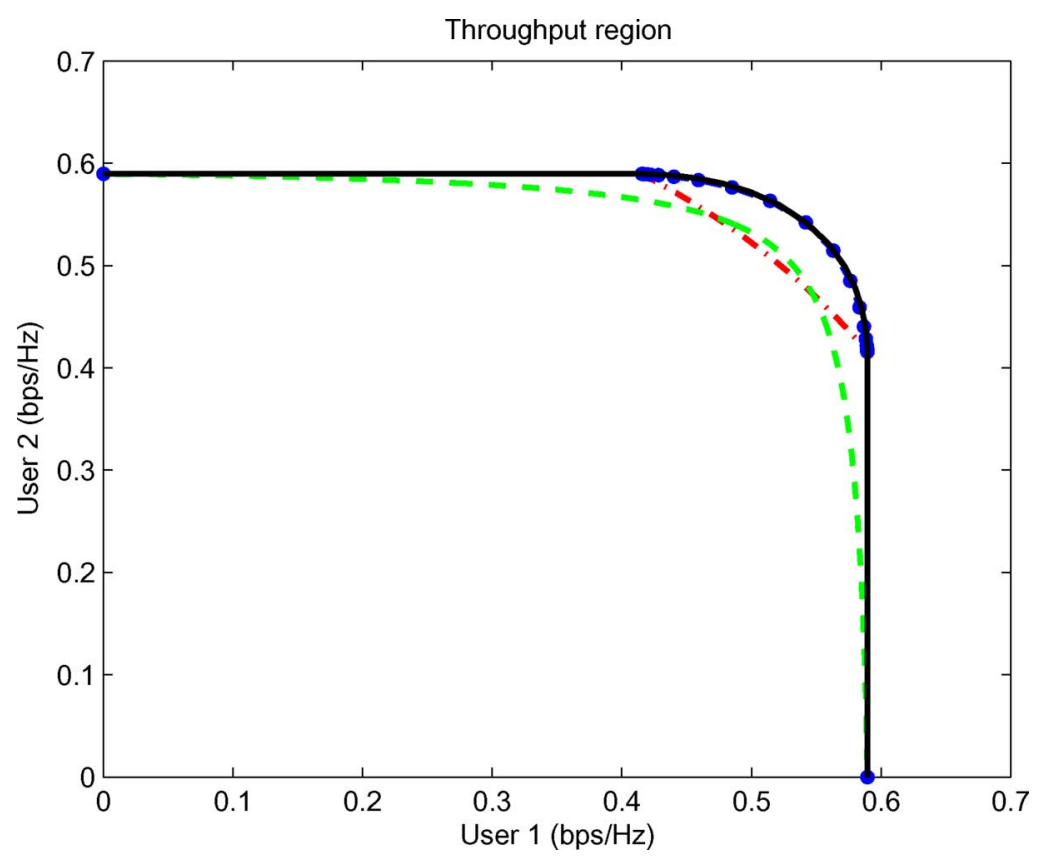

Fig. 2. Throughput region of two-user MAC case. $\mathrm{SNR}_{1}=\mathrm{SNR}_{2}=0 \mathrm{~dB} \cdot \theta_{1}=\theta_{2}=0.01$. The solid, dotted, dot-dashed, and dashed lines represent the regions achieved with optimal variable decoding order, suboptimal variable decoding order, fixed decoding with time sharing, and the TDMA, respectively.

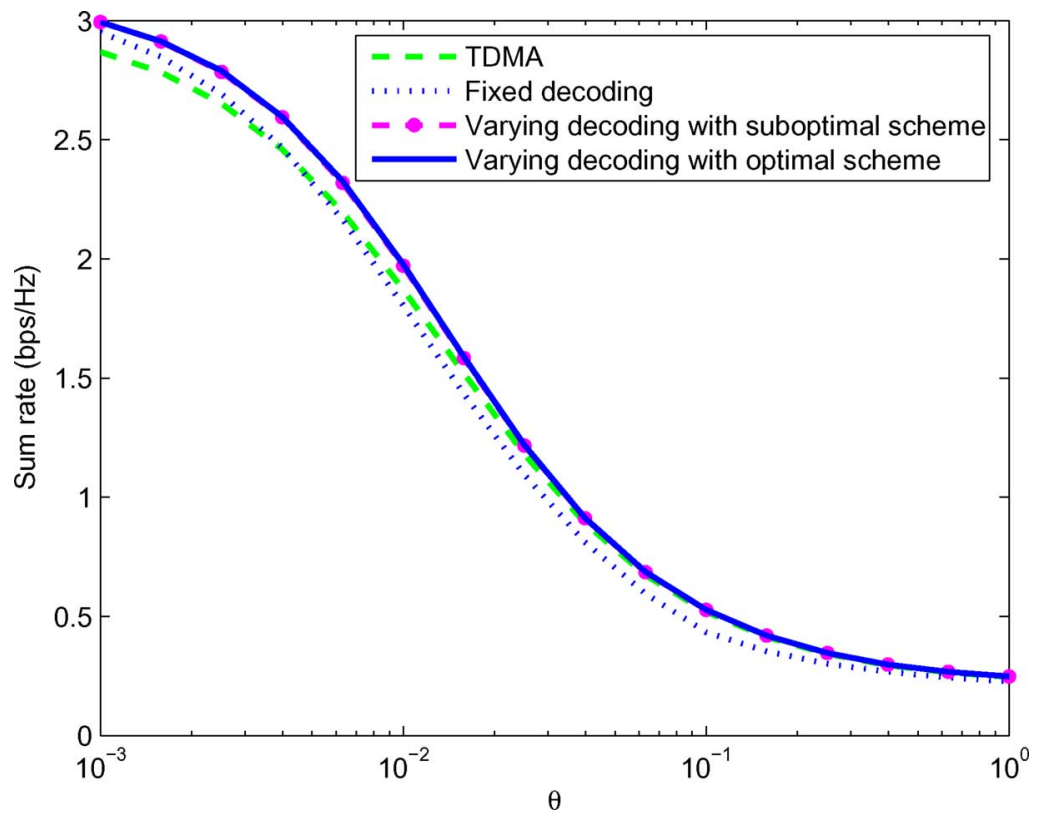

Fig. 3. Sum-rate throughput as a function of $\theta . \mathrm{SNR}_{1}=10 \mathrm{~dB} ; \mathrm{SNR}_{2}=0 \mathrm{~dB}$.

to the channel gains can significantly increase the achievable region. Finally, the throughput region of TDMA is given by the dashed curve. We immediately note that TDMA can achieve some points outside of the throughput region attained with fixed decoding order at the receiver side. These numerical results show that markedly different strategies may need to be employed when systems are operating under buffer constraints. In the absence of such constraints, the performance is captured by the ergodic capacity region which cannot be improved by varying the decoding order with respect to the channel states [6]. Hence, using a fixed decoding order at the receiver is an optimal strategy when there are no QoS constraints. Moreover, TDMA is always suboptimal with respect to the superposition schemes regardless of the decoding-order strategy [7].
In Fig. 3, sum-rate throughput, i.e., the sum of the effective capacities, is plotted as a function of the QoS exponent $\theta$. Here, we note that as $\theta$ increases, the curves of different strategies converge. In particular, TDMA performance approaches that of the superposition coding with variable decoding. Hence, orthogonal transmission strategies start being efficient in terms of attaining the sum rate under stringent buffer constraints. Note that the sum-rate throughput generally decreases with increasing $\theta$, and we conclude from the figure that this diminished throughput can be captured by having each user concentrate its power in a certain fraction of time in the TDMA scheme. We also see that for approximately $\theta>0.006$, TDMA starts outperforming superposition transmission when a fixed decoding order is employed at the receiver. Such an observation is also noted in the discus- 


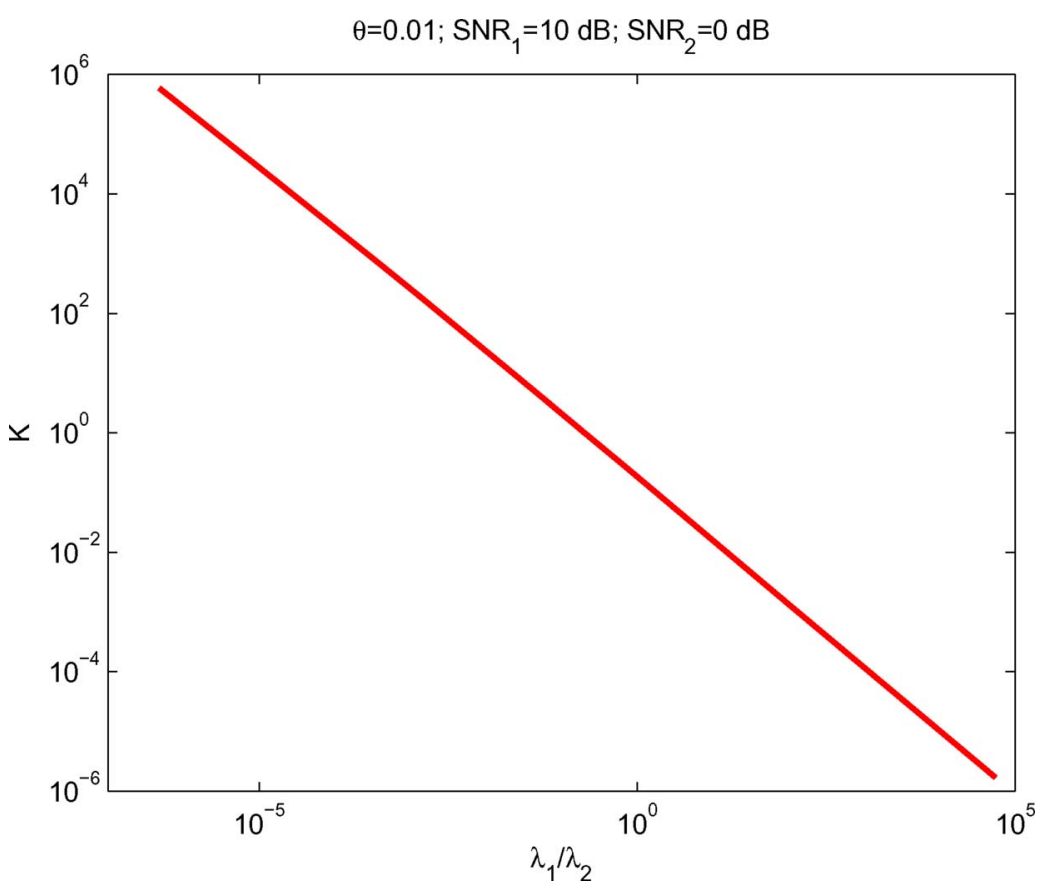

Fig. 4. $K$ versus $\frac{\lambda_{1}}{\lambda_{2}} \cdot \mathrm{SNR}_{1}=10 \mathrm{~dB}$. $\mathrm{SNR}_{2}=0 \mathrm{~dB} \cdot \theta_{1}=\theta_{2}=0.01$.

sion of Fig. 2. In contrast, we observe that as $\theta$ approaches 0 and hence the QoS constraints relax, TDMA is the strategy with the worst performance. Note that when the performance metric is the ergodic capacity and hence no queueing constraints are considered, this suboptimality of TDMA with respect to superposition strategies is well known (see, e.g., [7]).

We are also interested in the values of parameter $K$ that appears in the functions in Theorem 3. In Fig. 4, we plot $K$ as a function of $\frac{\lambda_{1}}{\lambda_{2}}=\frac{\lambda_{1}}{1-\lambda_{1}}$. It is interesting to note that $\log _{e} K$ seems to be linear with respect to $\log _{e}\left(\frac{\lambda_{1}}{1-\lambda_{1}}\right)$.

\section{TRANSMissions With POWER CONTROL}

In this section, we analyze the case in which the transmitter employs power control policies in the transmission. Similarly as previously, we initially investigate the scenario in which the decoding order is fixed for all channel states. Subsequently, we study variable decoding-order schemes. Note that varying the decoding order with respect to the channel states, according to the analysis in Section IV, has the potential to significantly affect the achievable rates.

\section{A. Power Control Policy for Fixed Decoding Order in All Channel States}

Here, we characterize the optimal power allocation policies when the decoding order is fixed for all channel states. Due to the convexity of $\mathcal{C}_{\text {MAC }}$, there exist Lagrange multipliers $\kappa=$ $\left(\kappa_{1}, \ldots, \kappa_{M}\right) \in \mathfrak{R}_{+}^{M}$ such that $\mathrm{C}^{*}(\Theta)$ on the boundary surface can be obtained by solving the optimization problem

$$
\max _{\mu} \lambda \cdot \mathrm{C}(\Theta, \mu)-\kappa \cdot \mathbb{E}\{\mu\}
$$

where $\mu=\left(\mu_{1}, \ldots, \mu_{M}\right)$ represents the collection of the power control policies of all users, $\lambda=\left(\lambda_{1}, \ldots, \lambda_{M}\right)$ is the weight vector, and $\mathrm{C}(\Theta, \mu)=\left(\mathrm{C}_{1}\left(\theta_{1}, \mu\right), \ldots, \mathrm{C}_{M}\left(\theta_{M}, \mu\right)\right)$ is the vector of maximum effective capacities of the users for given decoding order and power allocation policies. Note that $\mu_{j}=\frac{P_{j}}{N_{0} B}$ (defined in Section II as the instantaneous transmitted SNR level) describes the power control policy of the $j$ th user. For a given permutation $\pi$ and set of power allocations $\mu$, $\mathrm{C}_{j}\left(\theta_{j}, \mu\right)$ is given by

$$
\begin{aligned}
& C_{j}\left(\theta_{j}, \mu\right)= \\
& -\frac{1}{\theta_{j} T B} \log _{e} \mathbb{E}\left\{e^{-\theta_{j} T B \log _{2}\left(1+\frac{\mu_{j} z_{j}}{1+\sum_{\pi^{-1}(i)>\pi^{-1}(j)}^{\mu_{i} z_{i}}}\right)}\right\} .
\end{aligned}
$$

Now, the optimization problem (46) can be rewritten as

$$
\begin{aligned}
& \max _{\mu} \sum_{j=1}^{M} \\
& \quad-\lambda_{j} \frac{1}{\theta_{j} T B} \log _{e} \mathbb{E}\left\{e^{-\theta_{j} T B \log _{2}\left(1+\frac{\mu_{j} z_{j}}{1+\sum_{\pi^{-1}(i)>\pi^{-1}(j)}^{\mu_{i} z_{i}}}\right)}\right\} \\
& \quad-\sum_{j=1}^{M} \kappa_{j} \mathbb{E}\left\{\mu_{j}\right\} .
\end{aligned}
$$

The following result identifies the optimal power adaptation policies that solve the aforementioned optimization problem.

Theorem 4: Assume that the receiver, for all channel states, decodes the users in a fixed order specified by the permutation 
$\pi$. Then, the optimal power allocation policies that solve the optimization problem in (48) are given by

$$
\begin{aligned}
\mu_{j}= & \frac{\left(1+\sum_{\pi^{-1}(i)>\pi^{-1}(j)} \mu_{i} z_{i}\right)^{\frac{\beta_{j}}{\beta_{j}+1}}}{\alpha_{j}^{\frac{1}{\beta_{j}+1}} z_{j}^{\frac{\beta_{j}}{\beta_{j}+1}}} \\
& \left.-\frac{1+\sum_{\pi^{-1}(i)>\pi^{-1}(j)} \mu_{i} z_{i}}{z_{j}}\right)^{+} \text {for } j=1,2, \ldots, M
\end{aligned}
$$

where $\beta_{j}=\frac{\theta_{j} T B}{\log _{e} 2}$ is the normalized QoS exponent, $(x)^{+}=$ $\max \{x, 0\}$, and $\left(\alpha_{1}, \ldots, \alpha_{M}\right)$ are constants that are introduced to satisfy the average power constraints.

Proof: Note that with a fixed decoding order, the user $\pi(M)$ sees no interference from the other users, and hence, the derivative of (48) with respect to $\mu_{\pi(M)}$ will only be related to the effective capacity formulation of user $\pi(M)$. Therefore, we can solve an equivalent problem by maximizing $\mathrm{C}_{\pi(M)}$ instead. After we derive $\mu_{\pi(M)}$, the derivative of (48) with respect to $\mu_{\pi(M-1)}$ will only be related to the effective capacity formulation of user $\pi(M-1)$. By repeated application of this procedure, for given $\lambda$, (48) can be further decomposed into the following $M$ sequential optimization problems:

$$
\begin{aligned}
\max _{\mu_{j}}-\lambda_{j} \frac{1}{\theta_{j} T B} \log _{e} \mathbb{E}\left\{e^{-\theta_{j} T B \log _{2}\left(1+\frac{\mu_{j} z_{j}}{1+\sum_{\pi^{-1}(i)>\pi^{-1}(j)}^{\mu_{i} z_{i}}}\right)}\right\} \\
-\kappa_{j} \mathbb{E}\left\{\mu_{j}\right\}, \quad j \in\{1, \ldots, M\} \quad \text { (50) }
\end{aligned}
$$

in the inverse order of $\pi$. Similarly as in [15], due to the monotonicity of the logarithm, solving the aforementioned $M$ optimizations is the same as solving

$\min _{\mu_{j}} \mathbb{E}\left\{e^{-\theta_{j} T B \log _{2}\left(1+\frac{\mu_{j} z_{j}}{1+\sum_{\pi^{-1}(i)>\pi^{-1}(j)} \mu_{i} z_{i}}\right)}\right\}+\kappa_{j} \mathbb{E}\left\{\mu_{j}\right\}$

for $j \in\{1, \ldots, M\}$. Differentiating the aforementioned Lagrangian with respect to $\mu_{j}$ and setting the derivative to zero yield the intended result in (49).

Remark 6: Exploiting the result in (49), we can find that instead of adapting the power according to only its channel state as in [15], where a single-user scenario is studied, the user adapts the power with respect to its channel state normalized by the observed interference and the noise.

Remark 7: To give an explicit idea of the power control policy, we consider a two-user example in which the decoding order is $(2,1)$. For this case, we can easily find that

$$
\mu_{1}= \begin{cases}\frac{1}{\frac{1}{\beta_{1}+1}} z_{1}^{\frac{\beta_{1}}{\beta_{1}+1}}-\frac{1}{z_{1}}, & z_{1}>\alpha_{1} \\ 0, & \text { otherwise }\end{cases}
$$

and

$$
\begin{aligned}
& \mu_{2}= \\
& \left\{\begin{array}{lc}
\frac{1}{\alpha_{2}^{\frac{1}{\beta_{2}+1}} \frac{\beta_{2}}{\beta_{2}+1}}-\frac{1}{z_{2}}, & z_{1} \leq \alpha_{1} \\
\frac{\left(\frac{z_{1}}{\alpha_{1}}\right)^{\frac{\beta_{2}}{\left(\beta_{1}+1\right)\left(\beta_{2}+1\right)}}}{\alpha_{2}^{\frac{1}{\beta_{2}+1}} z_{2}^{\frac{\beta_{2}}{\beta_{2}+1}}}-\frac{\left(\frac{z_{1}}{\alpha_{1}}\right)^{\frac{1}{\beta_{1}+1}}}{z_{2}}, & z_{1}>\alpha_{1} \\
0, & \text { and } z_{2}>\alpha_{2} \\
0, & \text { otherwise } \frac{z_{2}}{\alpha_{2}}>\left(\frac{z_{1}}{\alpha_{1}}\right)^{\frac{1}{\beta_{1}+1}}
\end{array}\right.
\end{aligned}
$$

where $\alpha_{1}$ and $\alpha_{2}$ are chosen to satisfy the average power constraints of the two users.

\section{B. Power Control Policy for Variable Decoding Order}

In this section, we study the optimal power allocation policy when the receiver varies the decoding order with respect to the channel fading states. We mainly concentrate on the two-user scenario. The key idea we introduce here is to consider the power allocation policy of each user $j$ for each region $\mathcal{Z}_{m}$ (in which decoding is performed according to permutation $\pi_{m}$ ) while requiring the average power constraint to be satisfied by the joint power over all regions $\left\{\mathcal{Z}_{m}\right\}$.

For the two-user case, due to the convexity of the throughput region, there exist Lagrange multipliers $\kappa=\left(\kappa_{1}, \kappa_{2}\right) \in \mathfrak{R}_{+}^{2}$ such that $C^{*}(\Theta)$ on the boundary surface can be obtained by solving the optimization problem

$$
\max _{\mu} \lambda_{1} C_{1}(\mu, \mathcal{Z})+\lambda_{2} C_{2}(\mu, \mathcal{Z})-\kappa_{1} \mathbb{E}\left\{\mu_{1}\right\}-\kappa_{2} \mathbb{E}\left\{\mu_{2}\right\}
$$

where $\mu=\left(\mu_{1}, \mu_{2}\right)$ are the power control policies, $\left(\lambda_{1}, \lambda_{2}\right)$ are the weights in the weighted sum, and $\mathcal{Z}=\left(\mathcal{Z}_{1}, \mathcal{Z}_{2}\right)$ denotes a particular partition of the space of the positive values of $\mathbf{z}=$ $\left(z_{1}, z_{2}\right)^{1}$. Hence, power control policies that solve (54) are the optimal ones for a given partition. In the following, since we assume $\mathcal{Z}$ is given, the notation $C_{j}(\mu, \mathcal{Z})$ is replaced by $C_{j}(\mu)$ for brevity.

Recalling the discussion in Section IV-B, we can express the effective capacities of the two users as in (30) and (31) by only replacing $\mathrm{SNR}_{j}$ with $\mu_{j}(\mathbf{z})$ in these expressions. The Lagrangian [which is the objective function in (54)] can now be expressed as in (55), shown at the bottom of the next page.

In (55), the expressions in regions $\mathcal{Z}_{1}$ and $\mathcal{Z}_{2}$ are written separately due to the reason that possibly different power allocation strategies are employed in different regions. We define

$$
\begin{aligned}
\phi_{1}= & \int_{\mathbf{z} \in \mathcal{Z}_{1}}\left(1+\frac{\mu_{1} z_{1}}{1+\mu_{2} z_{2}}\right)^{-\beta_{1}} p_{\mathbf{z}}\left(z_{1}, z_{2}\right) d z_{1} d z_{2} \\
& +\int_{\mathbf{z} \in \mathcal{Z}_{2}}\left(1+\mu_{1} z_{1}\right)^{-\beta_{1}} p_{\mathbf{z}}\left(z_{1}, z_{2}\right) d z_{1} d z_{2}
\end{aligned}
$$

${ }^{1}$ Similarly as discussed in Section IV-B, different decoding orders are employed in $\mathcal{Z}_{1}$ and $\mathcal{Z}_{2}$. 
and

$$
\begin{aligned}
\phi_{2}= & \int_{\mathbf{z} \in \mathcal{Z}_{2}}\left(1+\frac{\mu_{2} z_{2}}{1+\mu_{1} z_{1}}\right)^{-\beta_{2}} p_{\mathbf{z}}\left(z_{1}, z_{2}\right) d z_{1} d z_{2} \\
& +\int_{\mathbf{z} \in \mathcal{Z}_{1}}\left(1+\mu_{2} z_{2}\right)^{-\beta_{2}} p_{\mathbf{z}}\left(z_{1}, z_{2}\right) d z_{1} d z_{2} .
\end{aligned}
$$

Note that the values of these functions are obtained for given power control policies $\mu=\left(\mu_{1}, \mu_{2}\right)$ and given partition $\mathcal{Z}=$ $\left(\mathcal{Z}_{1}, \mathcal{Z}_{2}\right)$.

Now, we consider the power control policy of each user in each decoding-order region $\mathcal{Z}_{i}, i=1,2$. By differentiating the Lagrangian, we can find the following optimality conditions:

1) $\frac{\lambda_{1}}{\phi_{1} \log _{e} 2}\left(1+\mu_{1} z_{1}\right)^{-\beta_{1}-1} z_{1}$

$$
-\frac{\lambda_{2}}{\phi_{2} \log _{e} 2}\left(1+\frac{\mu_{2} z_{2}}{1+\mu_{1} z_{1}}\right)^{-\beta_{2}-1} \frac{\mu_{2} z_{2} z_{1}}{\left(1+\mu_{1} z_{1}\right)^{2}}
$$$$
-\kappa_{1}=0 \quad \forall \mathbf{z} \in \mathcal{Z}_{1}
$$

2) $\frac{\lambda_{2}}{\phi_{2} \log _{e} 2}\left(1+\frac{\mu_{2} z_{2}}{1+\mu_{1} z_{1}}\right)^{-\beta_{2}-1} \frac{z_{2}}{1+\mu_{1} z_{1}}-\kappa_{2}=0$ $\forall \mathbf{z} \in \mathcal{Z}_{1}$ 3) $\frac{\lambda_{1}}{\phi_{1} \log _{e} 2}\left(\begin{array}{c}\left(1+\frac{\mu_{1} z_{1}}{1+\mu_{2} z_{2}}\right)^{-\beta_{1}-1} \frac{z_{1}}{1+\mu_{2} z_{2}}-\kappa_{1}=0 \\ \forall \mathbf{z} \in \mathcal{Z}_{2}\end{array}\right.$

$$
\begin{aligned}
4) & -\frac{\lambda_{1}}{\phi_{1} \log _{e} 2}\left(1+\frac{\mu_{1} z_{1}}{1+\mu_{2} z_{2}}\right)^{-\beta_{1}-1} \frac{\mu_{1} z_{1} z_{2}}{\left(1+\mu_{2} z_{2}\right)^{2}} \\
+ & \frac{\lambda_{2}}{\phi_{2} \log _{e} 2}\left(1+\mu_{2} z_{2}\right)^{-\beta_{2}-1} z_{2}-\kappa_{2}=0 \quad \forall \mathbf{z} \in \mathcal{Z}_{2}
\end{aligned}
$$

where (58) and (59) are obtained by differentiating the Lagrangian with respect to $\mu_{1}$ and $\mu_{2}$, respectively, over $\mathbf{z} \in \mathcal{Z}_{1}$. Similarly, (60) and (61) are obtained by differentiating with respect to $\mu_{1}$ and $\mu_{2}$, respectively, over $\mathbf{z} \in \mathcal{Z}_{2}$. Due to the convexity, whenever $\mu_{i}, i=1,2$ is negative valued, we set $\mu_{i}=0, i=1,2$. Although obtaining closed-form expressions from the optimality conditions seems to be unlikely, we can gather several insights on the power control policies by analyzing (58)-(61).

Let us first define $\alpha_{1}=\frac{\kappa_{1} \phi_{1} \log _{e} 2}{\lambda_{1}}, \alpha_{2}=\frac{\kappa_{2} \phi_{2} \log _{e} 2}{\lambda_{2}}, \alpha_{12}=$ $\frac{\kappa_{2} \phi_{1} \log _{e} 2}{\lambda_{1}}$, and $\alpha_{21}=\frac{\kappa_{1} \phi_{2} \log _{e} 2}{\lambda_{2}}$, where $\kappa_{1}, \kappa_{2}$ are the Lagrange multipliers whose values are chosen to satisfy the average power constraint (7) with equality, and $\phi_{1}$ and $\phi_{2}$ are defined in (56) and (57). Now, consider (58) and (59). The channel state lies in $\mathcal{Z}_{1}$. Through a simple computation using (59), we can derive

$$
\mu_{2}=\frac{\left(1+\mu_{1} z_{1}\right)^{\frac{\beta_{2}}{\beta_{2}+1}}}{\alpha_{2}^{\frac{1}{\beta_{2}+1}} z_{2}^{\frac{\beta_{2}}{\beta_{2}+1}}}-\frac{1+\mu_{1} z_{1}}{z_{2}}
$$

which tells us that $\mu_{2}=0$ if

$$
\frac{z_{2}}{1+\mu_{1} z_{1}}<\alpha_{2}
$$

If $\mu_{2}=0$, we have from (58) that

$$
\frac{\lambda_{1}}{\phi_{1} \log _{e} 2}\left(1+\mu_{1} z_{1}\right)^{-\beta_{1}-1} z_{1}-\kappa_{1}=0
$$

which gives us that

$$
\mu_{1}=\frac{1}{\alpha_{1}^{\frac{1}{\beta_{1}+1}} z_{1}^{\frac{\beta_{1}}{\beta_{1}+1}}}-\frac{1}{z_{1}}
$$

which implies that $\mu_{1}=0$ if

$$
z_{1}<\alpha_{1} .
$$

Now, if we substitute (62) into (58), we obtain the following additional condition for having $\mu_{1}=0$ : the equation

$$
\begin{aligned}
& \frac{z_{1}}{\alpha_{1}}\left(1+\mu_{1} z_{1}\right)^{-\left(\beta_{1}+1\right)} \\
& -\frac{z_{1} \alpha_{2}}{z_{2} \alpha_{12}}\left(\left(\frac{z_{2}}{\alpha_{2}\left(1+\mu_{1} z_{1}\right)}\right)^{\frac{1}{\beta_{2}+1}}-1\right)-1=0
\end{aligned}
$$

has a solution that returns a negative or zero value for $\mu_{1}$. The previous discussion enables us to characterize the regions in which one user transmits while the other one is silent. We also have a closed-form formula in (65) for the optimal power adaptation policy when only one user transmits. Indeed, this is the optimal power control policy derived in [15] for a single-user system. When both users transmit, the power control policies $\left(\mu_{1}, \mu_{2}\right)$ are given directly by the nonnegative solution of (58) and (59).

Note that the conditions and characterizations provided in (62)-(67) pertain to the case in which the channel state is in region $\mathcal{Z}_{1}$. Following a similar analysis of (60) and (61), we can obtain similar results for the cases in which the channel state is in $\mathcal{Z}_{2}$.

$$
\begin{aligned}
& \mathcal{J}=-\frac{\lambda_{1}}{\beta_{1} \log _{e} 2} \log _{e}\left(\int_{\mathbf{z} \in \mathcal{Z}_{1}}\left(1+\frac{\mu_{1} z_{1}}{1+\mu_{2} z_{2}}\right)^{-\beta_{1}} p_{\mathbf{z}}\left(z_{1}, z_{2}\right) d z_{1} d z_{2}+\int_{\mathbf{z} \in \mathcal{Z}_{2}}\left(1+\mu_{1} z_{1}\right)^{-\beta_{1}} p_{\mathbf{z}}\left(z_{1}, z_{2}\right) d z_{1} d z_{2}\right) \\
& -\frac{\lambda_{2}}{\beta_{2} \log _{e} 2} \log _{e}\left(\int_{\mathbf{z} \in \mathcal{Z}_{2}}\left(1+\frac{\mu_{2} z_{2}}{1+\mu_{1} z_{1}}\right)^{-\beta_{2}} p_{\mathbf{z}}\left(z_{1}, z_{2}\right) d z_{1} d z_{2}+\int_{\mathbf{z} \in \mathcal{Z}_{1}}\left(1+\mu_{2} z_{2}\right)^{-\beta_{2}} p_{\mathbf{z}}\left(z_{1}, z_{2}\right) d z_{1} d z_{2}\right) \\
& -\kappa_{1}\left(\mathbb{E}_{\mathbf{z} \in \mathcal{Z}_{1}}\left\{\mu_{1}\right\}+\mathbb{E}_{\mathbf{z} \in \mathcal{Z}_{2}}\left\{\mu_{1}\right\}\right)-\kappa_{2}\left(\mathbb{E}_{\mathbf{z} \in \mathcal{Z}_{1}}\left\{\mu_{2}\right\}+\mathbb{E}_{\mathbf{z} \in \mathcal{Z}_{2}}\left\{\mu_{2}\right\}\right)
\end{aligned}
$$


For a given partition $\left\{\mathcal{Z}_{1}, \mathcal{Z}_{2}\right\}$, the optimal power control policy can be determined numerically using the optimality conditions in (58)-(61). Additionally, the equations and inequalities in (62)-(67) can be used to guide the numerical algorithms as they specify under which conditions at most one user transmits and provide the optimal power control policy in such cases. However, there is one difficulty. Equations (62)-(67) depend on $\alpha_{1}, \alpha_{2}, \alpha_{12}$, and $\alpha_{21}$ which in turn depend on $\phi_{1}, \phi_{2}, \kappa_{1}$, and $\kappa_{2}$ which are in general functions of the power control policies. In such a situation, the following iterative procedure can be employed in search of the solution. We can first choose certain values for $\phi_{1}, \phi_{2}, \kappa_{1}$, and $\kappa_{2}$, and then determine the optimal power allocation policies for these selected values. Subsequently, we can check whether the obtained policy satisfies the average power constraint with equality. This enables us to determine if the selected $\kappa_{1}$ and $\kappa_{2}$ values are accurate. We can also compute $\phi_{1}$ and $\phi_{2}$ using the obtained policy and see if they agree with the initial values of $\phi_{1}$ and $\phi_{2}$. If there is no sufficient match or if the power constraint is not satisfied with equality, then we update the values of $\phi_{1}, \phi_{2}, \kappa_{1}$, and $\kappa_{2}$, and reiterate the search of the optimal policy.

With this insight, we propose the following algorithm that can be used to determine the optimal power allocated to each channel state.

\section{POWER CONTROL ALGORITHM}

1 Given $\lambda_{1}, \lambda_{2}$, the partition $\mathcal{Z}$, initialize $\phi_{1}, \phi_{2}$;

2 Initialize $\kappa_{1}$ and $\kappa_{2}$;

3 Determine $\alpha_{1}=\frac{\kappa_{1} \phi_{1} \log _{e} 2}{\lambda_{1}}, \alpha_{2}=\frac{\kappa_{2} \phi_{2} \log _{e} 2}{\lambda_{2}}$, $\alpha_{12}=\frac{\kappa_{2} \phi_{1} \log _{e} 2}{\lambda_{1}}, \alpha_{21}=\frac{\kappa_{1} \phi_{2} \log _{e} 2}{\lambda_{2}}$;

4 if $\mathbf{z} \in \mathcal{Z}_{1}$

5 then if $z_{2}>\alpha_{2}$

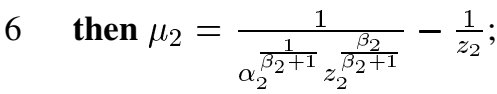

7 if $\frac{z_{1}}{\alpha_{1}}\left(1+\mu_{1} z_{1}\right)^{-\left(\beta_{1}+1\right)}-$ $\frac{z_{1} \alpha_{2}}{z_{2} \alpha_{21}}\left(\left(\frac{z_{2}}{\alpha_{2}\left(1+\mu_{1} z_{1}\right)}\right)^{\frac{1}{\beta_{2}+1}}-1\right)-1=0$ returns nonpositive $\mu_{1}$

$8 \quad$ then $\mu_{1}=0$

$9 \quad$ else if $\frac{z_{2}}{\alpha_{2}}<\left(\frac{z_{1}}{\alpha_{1}}\right)^{\frac{1}{\beta_{1}+1}}$

$$
\text { then } \mu_{2}=0, \mu_{1}=\left[\frac{1}{\frac{1}{\alpha_{1}^{\beta_{1}+1}} z_{1}^{\beta_{1}+1}}-\frac{1}{z_{1}}\right]^{+} \text {; }
$$

11 else Compute $\mu_{1}, \mu_{2}$ from (58) and (59);

else $\mu_{2}=0, \mu_{1}=\left[\frac{1}{\alpha_{1}^{\frac{\beta_{1}+1}{\beta_{1}}} z_{1}^{\frac{\beta_{1}+1}{1}}}-\frac{1}{z_{1}}\right]^{+}$;

13 if $\mathbf{z} \in \mathcal{Z}_{2}$

14 then if $z_{1}>\alpha_{1}$
15 then $\mu_{1}=\frac{1}{\alpha_{1}^{\frac{1}{\beta_{1}+1}} z_{1}^{\frac{\beta_{1}}{\beta_{1}+1}}}-\frac{1}{z_{1}}$;

16 if $\frac{z_{2}}{\alpha_{2}}\left(1+\mu_{2} z_{2}\right)^{-\left(\beta_{2}+1\right)}-$

$\frac{z_{2} \alpha_{1}}{z_{1} \alpha_{21}}\left(\left(\frac{z_{1}}{\alpha_{1}\left(1+\mu_{2} z_{2}\right)}\right)^{\frac{1}{\beta_{1}+1}}-1\right)-1=0$ returns

nonpositive $\mu_{2}$

17 then $\mu_{2}=0$;

18 else if $\frac{z_{1}}{\alpha_{1}}<\left(\frac{z_{2}}{\alpha_{2}}\right)^{\frac{1}{\beta_{2}+1}}$

19

then $\mu_{1}=0, \mu_{2}=\left[\frac{1}{\alpha_{2}^{\frac{\beta_{2}+1}{2}} z_{2}^{\frac{\beta_{2}}{\beta_{2}+1}}}-\frac{1}{z_{2}}\right]^{+}$;

20 else Compute $\mu_{1}, \mu_{2}$ from (60) and (61);

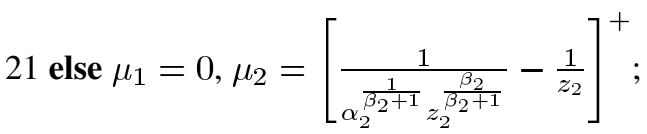

22 Check if the obtained power control policies $\mu_{1}$ and $\mu_{2}$ satisfy the power constraint with equality;

23 if not satisfied with equality

24 then update the values of $\kappa_{1}$ and $\kappa_{2}$ and return to Step 3;

25 else move to Step 26;

26 Evaluate $\phi_{1}$ and $\phi_{2}$ with the obtained power control policies;

27 Check if the new values of $\phi_{1}$ and $\phi_{2}$ agree (up to a certain margin) with those used in Step 3;

28 if do not agree

29 then update the values of $\phi_{1}$ and $\phi_{2}$ and return to Step 2;

30 else declare the obtained power allocation policies $\mu_{1}$ and $\mu_{2}$ as the optimal ones.

Note that, earlier, we have not specified how the values of $\kappa_{1}, \kappa_{2}, \phi_{1}$, and $\phi_{2}$ are updated for each iteration in order to keep the algorithm generic. In our numerical computations, we have updated $\kappa_{1}$ and $\kappa_{2}$ using the bisection search algorithm. The values of $\phi_{1}$ and $\phi_{2}$ are updated in Step 29 of the algorithm by assigning them the values evaluated in Step 26. Hence, the most recent values are carried over to the new iteration.

In Fig. 5, we plot the optimal power allocation policies $\mu_{1}$ and $\mu_{2}$ as functions of channel fading states $z_{1}$ and $z_{2}$. We assume that $\theta_{1}=\theta_{2}=0.01, \mathrm{SNR}_{1}=\mathrm{SNR}_{2}=0 \mathrm{~dB}$, and $\lambda_{1}=$ $\lambda_{2}=0.5$. We consider the partition specified by the suboptimal decoding order given in (43). Hence, since we have $\lambda_{1}=\lambda_{2}=$ 0.5 , decoding orders $(1,2)$ and $(2,1)$ are used when $z_{2}<z_{1}$ and $z_{2}>z_{1}$, respectively. Under these assumptions, we computed the optimal values as $\kappa_{1}^{*}=0.0470, \kappa_{2}^{*}=0.0462, \phi_{1}^{*}=0.5550$, and $\phi_{2}^{*}=0.5538$. In the figure, we observe that each user, not surprisingly, allocates most of its power to the regions in which it is decoded last and hence does not experience interference. However, due to the introduction of QoS constraints, we also note that each user also allocates certain power to the cases in which it is decoded first. This is performed in order to continue transmission and avoid buffer overflows. 


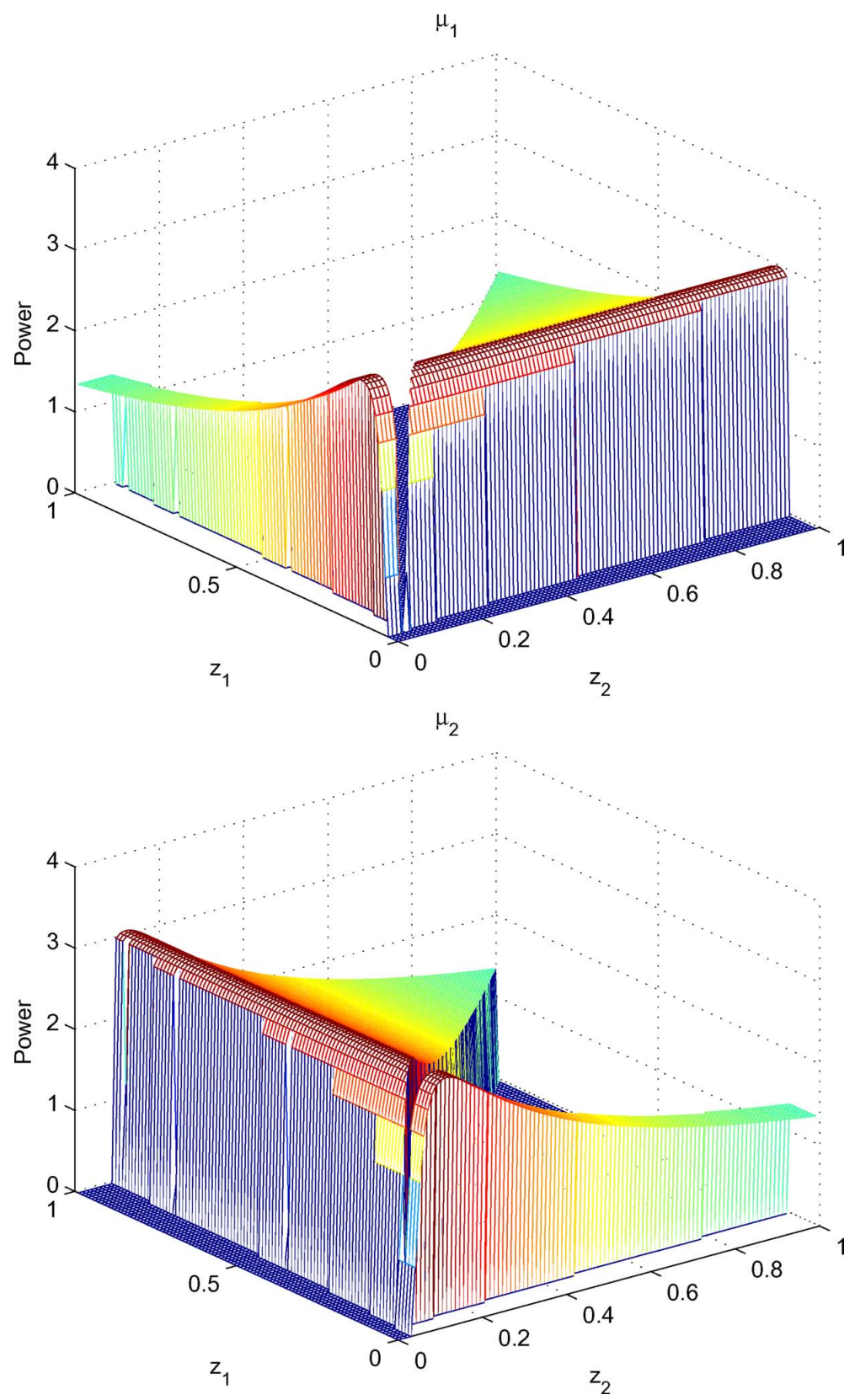

Fig. 5. Optimal power control policies $\mu_{1}$ and $\mu_{1}$ of users 1 and 2 , respectively, as a function of $\left(z_{1}, z_{2}\right) . \lambda_{1}=0.5, \lambda_{2}=0.5$.

So far, we have assumed that the partition $\mathcal{Z}$ is given. The optimal partition $\mathcal{Z}$ that maximizes the weighted sum-rate can be derived through the following optimization similarly as in [32]:

$$
\mathrm{C}^{*}=\sup _{\mathcal{Z}} \lambda_{1} \mathrm{C}_{1}(\mu, \mathcal{Z})+\lambda_{2} \mathrm{C}_{2}(\mu, \mathcal{Z})
$$

where $C^{*}$ is the optimal weighted sum value for given pair of $\left(\lambda_{1}, \lambda_{2}\right)$, and $\mu=\left(\mu_{1}, \mu_{2}\right)$ are the optimal power control policies for given $\mathcal{Z}$.

\section{CONCLUSION}

In this paper, we have studied the achievable throughput regions in multiple-access fading channels when users operate under QoS constraints. We have assumed that both the transmitters and the receiver have perfect CSI. We have employed the effective capacity as a measure of the throughput under buffer constraints. We have defined the effective capacity region and shown its convexity. We have considered different transmission and reception scenarios e.g., superposition coding, different strategies for the decoding order, and TDMA. When transmission with superposition coding is performed, we have shown that varying the decoding order at the receiver with respect to the fading states can significantly increase the achievable rate region compared to that achieved with fixed decoding-order schemes. For the case of two users with the same QoS constraints, we have derived the optimal strategy for varying the decoding order. We have noted that when the two users operate under similar QoS limitations and one user enjoys much more favorable channel conditions, the efficient strategy is to first decode the user with 
the better channel and subsequently decode the other user so that the user with worse channel conditions does not experience interference. Motivated by this observation, we have proposed for general multiple-user scenarios a simpler suboptimal decoding rule which can almost perfectly match the optimal throughput region. We have also studied the performance of orthogonal transmission strategies by considering TDMA. In the numerical results, we have demonstrated that TDMA can perform better than superposition coding with fixed decoding order for certain QoS constraints. More specifically, we have noted that TDMA can support arrival rate pairs that are strictly outside the region achieved when fixed decoding order is employed at the receiver. We have also observed that the performance of TDMA approaches that of the optimal strategy of superposition coding with variable decoding order as $\theta$ increases (i.e., as the QoS constraints become more stringent).

In the second part of this paper, we have incorporated power adaptation strategies into the model. For a given fixed decoding order at the receiver, we have identified the optimal power control policies. We have seen that the optimal schemes adapt the power by treating the observed interference as additional noise. Since the observed interference depends on the power control policies of the other users that will be decoded later, a coupling is introduced between the optimal policies. For cases in which a variable decoding-order strategy is adopted by the transmitter, we have obtained the conditions that the optimal strategies should satisfy and described an algorithm to achieve these optimal schemes.

\section{REFERENCES}

[1] R. Gallager, "An inequality on the capacity region of multiaccess fading channels," in Commun. Cryptography-Two Sides One Tapestry. New York: Springer, 1994, pp. 129-139.

[2] T. M. Cover and J. A. Thomas, Elements of Information Theory. New York: Wiley, 1991.

[3] D. Tse and S. Hanly, "Multi-access fading channels-Part I: Polymatroid structure, optimal resource allocation and throughput capacities," IEEE Trans. Inform. Theory, vol. 44, no. 7, pp. 2796-2815, Nov. 1998.

[4] R. Knopp and P. A. Humblet, "Information capacity and power control in single-cell multiuser communications," in Proc. IEEE Int. Conf. Commun., Seattle, WA, Jun. 1995, vol. 1, pp. 331-335.

[5] G. A. Gupta and S. Toumpis, "Power allocation over parallel Gaussian multiple access and broadcast channels," IEEE Trans. Inf. Theory, vol. 52, no. 7, pp. 3274-3282, Jul. 2006.

[6] S. Vishwanath, S. A. Jafar, and A. Goldsmith, "Optimum power and rate allocation strategies for multiple access fading channels," in Proc. IEEE Veh. Technol. Conf., 2001, pp. 2888-2892.

[7] G. Caire, D. Tuninetti, and S. Verdú, "Suboptimality of TDMA in the low-power regime," IEEE Trans. Inf. Theory, vol. 50, no. 4, pp. 608-620, Apr. 2004.

[8] E. M. Yeh and A. S. Cohen, "Information theory, queueing, and resource allocation in multi-user fading communications," in Proc. Conf. Inf. Sci. Syst., Princeton, NJ, Mar. 17-19, 2004, pp. 1396-1401.

[9] E. M. Yeh and A. S. Cohen, "Throughput optimal power and rate control for multiaccess and broadcast communications," in Proc. Int. Symp. Inf. Theory, Chicago, IL, Jun. 27--Jul. 2 2004, p. 112.

[10] E. M. Yeh and A. S. Cohen, "Throughput and delay optimal resource allocation in multiaccess fading channels," in Proc. 2003 Int. Symp. Inf. Theory, 2003, p. 245.

[11] E. M. Yeh and A. S. Cohen, "Delay optimal rate allocation in multiaccess fading communications," in Proc. Allerton Conf. Commun., Control, Comput., Monticello, IL, Sep. 29-Oct. 1 2004, pp. 140-149.
[12] N. Ehsan and T. Javidi, "Delay optimal transmission policy in a wireless multiaccess channel," IEEE Trans. Inf. Theory, vol. 54, no. 8, pp. 3745-3751, Aug. 2008.

[13] J. Yang and S. Ulukus, "Delay-minimal transmission for average power constrained multi-access communications," IEEE Trans. Wireless Commun., vol. 9, no. 9, pp. 2754-2767, Sep. 2010.

[14] D. Wu and R. Negi, "Effective capacity: A wireless link model for support of quality of service," IEEE Trans. Wireless Commun., vol. 2, no. 4, pp. 630-643, Jul. 2003.

[15] J. Tang and X. Zhang, "Quality-of-service driven power and rate adaptation over wireless links," IEEE Trans. Wireless Commun., vol. 6, no. 8, pp. 3058-3068, Aug. 2007.

[16] J. Tang and X. Zhang, "Cross-layer-model based adaptive resource allocation for statistical QoS guarantees in mobile wireless networks," IEEE Trans. Wireless Commun., vol. 7, no. 6, pp. 2318-2328, Jun. 2008.

[17] L. Liu, P. Parag, J. Tang, W.-Y. Chen, and J.-F. Chamberland, "Resource allocation and quality of service evaluation for wireless communication systems using fluid models," IEEE Trans. Inf. Theory, vol. 53, no. 5, pp. 1767-1777, May 2007.

[18] L. Liu, P. Parag, and J.-F. Chamberland, "Quality of service analysis for wireless user-cooperation networks," IEEE Trans. Inf. Theory, vol. 53, no. 10, pp. 3833-3842, Oct. 2007.

[19] L. Liu and J.-F. Chamberland, "On the effective capacities of multipleantenna Gaussian channels," in IEEE Int. Symp. Inf. Theory, Toronto, ON, Canada, 2008, pp. 2583-2587.

[20] M. C. Gursoy, D. Qiao, and S. Velipasalar, "Analysis of energy efficiency in fading channels under QoS constraints," IEEE Trans. Wireless Commun., vol. 8, no. 8, pp. 4252-4263, Aug. 2009.

[21] A. Balasubramanian and S. L. Miller, "On optimal scheduling for timedivision systems with quality of service constraints," in Proc. 43rd Annu. Conf. Inf. Sci. Syst., Mar. 2009, pp. 719-722.

[22] C.-S. Chang and T. Zajic, "Effective bandwidths of departure processes from queues with time varying capacities," in Proc. IEEE Infocom, 1995, pp. 1001-1009.

[23] M. C. Gursoy, "Throughput analysis of buffer-constrained wireless systems in the finite blocklength regime," presented at the IEEE Int. Conf. Commun., Kyoto, Japan, Jun. 2011.

[24] D. Qiao, M. C. Gursoy, and S. Velipasalar, "Channel coding over multiple coherence blocks with queueing constraints," presented at the IEEE Int. Conf. Commun., Kyoto, Japan, Jun. 2011.

[25] S. Shakkottai, "Effective capacity and QoS for wireless scheduling," IEEE Trans. Automat. Control, vol. 53, no. 3, pp. 749-761, Apr. 2008.

[26] V. J. Venkataramanan and X. Lin, "On wireless scheduling algorithms for minimizing the queue-overflow probability," IEEE/ACM Trans. Network., vol. 18, no. 3, pp. 788-801, Jun. 2010.

[27] L. Ying, R. Srikant, A. Eryilmaz, and G. Dullerud, "A large deviations analysis of scheduling in wireless networks," IEEE Trans. Inf. Theory, vol. 52, no. 11, pp. 5088-5098, Nov. 2008.

[28] A. Goldsmith, Wireless Communications, 1st ed. Cambridge, U.K.: Cambridge Univ. Press, 2005.

[29] S. Boyd and L. Vandenberghe, Convex Optimization. Cambridge, U.K.: Cambridge Univ. Press, 2004.

[30] G. B. Arfken, Mathmatical Methods for Physicist. New York: Academic, 1985

[31] C.-S. Chang, Performance Guarantees in Communication Networks. New York: Springer-Verlag, 2000.

[32] L. Lai and H. El Gamal, "The water-filling game in fading multiple-access channels," IEEE Trans. Inf. Theory, vol. 54, no. 5, pp. 2110-2122, May 2008.

Deli Qiao received the B.E. degree in electrical engineering from Harbin Institute of Technology, Harbin, China, in 2007. He is currently a research assistant working toward the Ph.D. degree in the Department of Electrical Engineering, University of Nebraska-Lincoln.. His research interests include information theory and wireless communications, with an emphasis on quality of service (QoS) provisioning. In 2011, he received the Maude Hammond Fling Fellowship from the University of Nebraska-Lincoln. 
Mustafa Cenk Gursoy received the Ph.D. degree in electrical engineering from Princeton University, Princeton, NJ, in 2004, and the B.S. degree in electrical and electronics engineering from Bogazici University, Istanbul, Turkey, in 1999 with high distinction. He was a recipient of the Gordon Wu Graduate Fellowship from Princeton University between 1999 and 2003. In the summer of 2000, he worked at Lucent Technologies, Holmdel, NJ, where he conducted performance analysis of DSL modems.

Between 2004 and 2011, he was a faculty member in the Department of Electrical Engineering at the University of Nebraska-Lincoln (UNL). He is currently an Associate Professor in the Department of Electrical Engineering and Computer Science at Syracuse University. His research interests are in the general areas of wireless communications, information theory, communication networks, and signal processing.

$\mathrm{He}$ is currently a member of the editorial boards of IEEE Transactions on Wireless Communications and Physical Communication (Elsevier). He received an NSF CAREER Award in 2006. More recently, he received the EURASIP Journal of Wireless Communications and Networking Best Paper Award, the UNL College Distinguished Teaching Award, and the Maude Hammond Fling Faculty Research Fellowship.
Senem Velipasalar received the Ph.D. and M.A. degrees in electrical engineering from Princeton University, Princeton, NJ, in 2007 and 2004, respectively, the M.S. degree in electrical sciences and computer engineering from Brown University, Providence, RI, in 2001 and the B.S. degree in electrical and electronic engineering with high honors from Bogazici University, Istanbul, Turkey, in 1999.

During the summers of 2001 to 2005, she was with the Exploratory Computer Vision Group at IBM T. J. Watson Research Center, NY. Between 2007 and 2011, she was an Assistant Professor in the Department of Electrical Engineering at the University of Nebraska-Lincoln. Currently, she is an Assistant Professor in the Department of Electrical Engineering and Computer Science at Syracuse University, Syracuse, NY. The focus of her research has been on wireless embedded smart cameras, multi-camera tracking and surveillance systems, and automatic event detection from videos. Her current research interests include embedded computer vision, video/image processing, embedded smart camera systems, distributed multicamera systems, pattern recognition, and signal processing.

Dr. Velipasalar received an NSF CAREER Award in 2011. She received the Best Student Paper Award at the IEEE International Conference on Multimedia and Expo in 2006. She is the recipient of the EPSCoR First Award, two Layman Awards, the IBM Patent Application Award, and the Princeton and Brown University Graduate Fellowships. 